\title{
Some Results of Deformations on Compact $H$-twisted Generalized Calabi-Yau Manifolds
}

\author{
KANG WEI
}

\begin{abstract}
In this paper, we prove several formulas related to Hodge theory, and using them to prove the deformations of a compact $H$-twisted generalized Calabi-Yau manifold are unobstructed and $L^{2}$ convergence in a fixed neighbourhood in another power series. And if we assume that the deformation is smooth in a fixed neighbourhood, and assume the existence of a global canonical family of deformation, we also construct the global canonical family of the deformations of generalized Kähler manifolds.

Keywords: Deformations of complex structures, Hodge theory, Hermitian and Kählerian manifolds, Calabi-Yau manifolds.
\end{abstract}

\section{Introduction}

The generalized complex geometry is introduced by N. Hitchin and developed by M. Gualtieri and G. R. Cavalcanti and many others in [2, 3, 5, 6, 10. This new geometry provides an indeed broad platform for the people working in both mathematics and physics. The theory of deformations of complex structures can be dated back to Riemann, and extensively studied by K. Kodaira, D. C. Spencer, N. Nirenberg, M. Kuranishi and many other great mathematicians in [11, 15]. The deformation theory of generalized complex geometry is first studied by M. Gualtieri, R. Goto and so on in in [2, 9]. The concept of $H$-twisted was introduced by P. Ševera and A. Weinstein in [16. Yi Li in [14] has proved that the deformations of a compact H-twisted Generalized Calabi-Yau manifold are unobstructed in an infinitesimal neighborhood by using Kodaira-Spencer-Kuranishis method.

Received January 11, 2015.

Mathematics Subject Classification. 2010 Primary 32G05; Secondary 58A14, $53 \mathrm{C} 55,14 \mathrm{~J} 32$. 
In this paper, we re-state the deformations of a compact $H$-twisted generalized Calabi-Yau manifold are unobstructed and $L^{2}$ convergence in a fixed neighbourhood by using Hodge theory. And if we assume that the deformation is smooth in a fixed neighbourhood, we also construct the global canonical family of the deformations by using the parallel method in [12].

This paper is organized as follows. In Section 2 and Section 3 , we introduce some basic definitions and some propositions of compact $H$-twisted generalized Calabi-Yau manifolds which we will use. In Section 4 , by using the Hodge theory, we prove the following two propositions which will be used in constructing the deformations.

Lemma 1.1. On compact generalized Kähler manifold $M$, For any $\rho \in$ $\wedge^{*} T_{M}^{*}$, we have

$$
\begin{aligned}
\left\|\bar{\partial}_{H}^{*} G \rho\right\|^{2} & \leq(\rho, G \rho), \\
\left\|\bar{\partial}_{H}^{*} G \partial_{H} \rho\right\|^{2} & \leq\|\rho\|^{2},
\end{aligned}
$$

where $\bar{\partial}_{H}^{*}$ is the adjoint operator of $\bar{\partial}_{H}, G$ is the Green operator corresponding to the harmonic operator $\triangle_{\bar{\partial}_{H}}, \bar{\partial}_{H}, \partial_{H}$ is definite in Definition 2.5 and Definition 2.10, and the norms $\|\cdot\|$ is definite in Definition 2.7 in Section 2 in details.

Proposition 1.2. Let $(M, J)$ be a compact $H$-twisted generalized Kähler manifold. Then for any $\rho \in \wedge^{*} T_{M}^{*}$,

$$
s=\bar{\partial}_{H}^{*} G \partial_{H} \rho
$$

is a solution to the equation $\bar{\partial}_{H} s=\partial_{H} \rho$ with condition $\bar{\partial}_{H} \partial_{H} \rho=0$, such that

$$
\|s\|^{2} \leq\left(\partial_{H} \rho, G \partial_{H} \rho\right)
$$

where $(\cdot, \cdot)$ is the Born-Infeld inner product definite in Definition 2.7. Moreover, if $\mathbb{H} s=0$ and $\bar{\partial}_{H}^{*} s=0, s$ is uniquely determined.

In Section 5, we re-state that the deformations of a compact $H$-twisted generalized Calabi-Yau manifold are unobstructed and $L^{2}$ convergence in a fixed neighbourhood by another power series. In details, we have 
Some Results of Deformations on Compact $H$-twisted Generalized...133

Theorem 1.3. Let $(M, J)$ be a compact $H$-twisted generalized Calabi-Yau manifold. Then there exists a globally $L^{2}$ convergent power series which determines the deformation in $t<\frac{1}{4 a c}$,

$$
\epsilon(t) \rho_{0}=\sum_{i=1}^{N} \epsilon_{i} \rho_{0} t^{i}+\sum_{k \geq 2} \sum_{k_{1}+\cdots+k_{N}=k, k_{i} \geq 0} \epsilon_{k_{1} \cdots k_{N}} \rho_{0}\left(t^{1}\right)^{k_{1}} \cdots\left(t^{N}\right)^{k_{N}},
$$

which satisfies:

(1) $\bar{\partial}_{H} \epsilon(t) \rho_{0}+\frac{1}{2}[\epsilon(t), \epsilon(t)]_{H} \rho_{0}=0$;

(2) $\epsilon_{k_{1} \cdots k_{N}} \rho_{0}$ is $\bar{\partial}_{H}^{*}$-closed, and $\partial_{H}$-exact for any $k_{1}+\cdots+k_{N}=k \geq 2$;

(3) $\epsilon(t) \rho_{0}$ is $L^{2}$ convergence in $t<\frac{1}{4 a c}$.

The convergence of the deformations is proved by using the power series in Lemma 3.4 as follows:

Lemma 1.4 (See Lemma 4.1 in [12]). Let $x_{1}=a$ be a constant, and $x_{k}=c \sum_{i=1}^{k-1} x_{i} x_{k-i}$, where $c$ is a constant, then $\sum_{i=1}^{\infty} x_{i} t^{i}$ converge on $|t| \leq$ $\frac{1}{4 a c}$.

In Section 6, if we assume the existence of a global canonical family of deformation, we also construct the global canonical family of the deformations of generalized Kähler manifolds. We have the theorem as follows:

Proposition 1.5. On compact generalized Kähler manifold $M$, if we assume that $\epsilon(t)$ smooth with convergence radius $\frac{1}{4 a c}$ exists. Then

$$
\epsilon(t) \rho_{0} \in H_{\bar{\partial}_{H}}^{2}\left(M, \wedge^{*} T_{M}^{*}\right),
$$

where $H_{\bar{\partial}_{H}}^{2}\left(M, \wedge^{*} T_{M}^{*}\right)$ is the cohomology group of the complex $\left(\wedge^{*} T_{M}^{*}, \bar{\partial}_{H}\right)$, and there exists

$$
\rho_{t}:=\rho_{0}+\sum_{K,|K| \geq 1} \rho_{K} t^{K} \in U_{0}
$$

where $t^{K}:=\left(t^{1}\right)^{k_{1}} \cdots\left(t^{N}\right)^{k_{N}},|K|:=k_{1}+\cdots+k_{N}$, such that

(1) $\rho_{t}^{c}:=e^{-\epsilon(t)} \rho_{t}$ holomorphic with respect to $J_{\epsilon(t)}$,

(2) $\rho_{K}$ is $\partial_{H}$-exact and $\bar{\partial}_{H}^{*}$-closed, $(|K| \geq 1)$

(3) $\rho_{t}$ converges with radius $\frac{1}{16 a^{2} c^{2}}$.

And we also give the representations of the global canonical family of the deformations: 
Proposition 1.6.

$$
\rho_{t}^{c}:=e^{-\epsilon(t)} \rho_{t}
$$

and

$$
\left[\rho_{t}^{c}\right]=\left[\rho_{0}\right]+\sum_{i=1}^{N}\left[\mathbb{H}\left(-\epsilon_{i} \rho_{0}\right)\right] t+O\left(|t|^{2}\right),
$$

where $\left[\rho_{0}\right]$ means a representation in $H_{\bar{\partial}_{H}}^{0}\left(M, \wedge^{*} T_{M}^{*}\right)$, and $O\left(|t|^{2}\right)$ denotes the terms in

$H_{\bar{\partial}_{H}}^{4}\left(M, \wedge^{*} T_{M}^{*}\right) \bigoplus \cdots \oplus H_{\bar{\partial}_{H}}^{2 n}\left(M, \wedge^{*} T_{M}^{*}\right)$ of order at least 2 in $t$.

The method we use is parallel to the method in [12].

Thanks to my supervisor Professor Kefeng Liu. Also thanks to Dr. Sheng Rao, Dr. Shengmao Zhu, Professor D. Baraglia, and Professor M. Gualtieri.

\section{Some basic definitions}

In this section, we review some basic definitions of compact $H$-twisted generalized Calabi-Yau manifolds. We refer the reader to [1-3, 8, 10] for details.

We define a pair on smooth manifold $M$ similar to the pair between $T_{M}$ and $T_{M}^{*}$.

Definition 2.1. Let $M^{n}$ be a smooth manifold with dim $_{\mathbb{R}}=n$. We define the following pair on $\left(T_{M} \oplus T_{M}^{*}\right) \otimes_{\mathbb{R}} \mathbb{C}$ :

$$
<A, B>\quad:=\quad<X+\xi, Y+\eta>:=\frac{1}{2}(\xi(Y)+\eta(X)),
$$

where $A:=X+\xi, B:=Y+\eta \in\left(T_{M} \oplus T_{M}^{*}\right) \otimes_{\mathbb{R}} \mathbb{C}, X, Y \in T_{M} \otimes_{\mathbb{R}} \mathbb{C}, \xi, \eta \in$ $T_{M}^{*} \otimes_{\mathbb{R}} \mathbb{C}$.

We can define the generalized complex structures similar to complex structures on $M$.

Definition 2.2. Let $M^{n}$ be a smooth manifold with $\operatorname{dim}_{\mathbb{R}}=n$. If there exists an endomorphism $J$ on $\left(T_{M} \oplus T_{M}^{*}\right) \otimes_{\mathbb{R}} \mathbb{C}$, satisfying 
Some Results of Deformations on Compact $H$-twisted Generalized...135

$$
J^{2}=-1
$$

and

$$
<A, B>=<J A, J B>
$$

$J$ is called a generalized almost complex structure on $M$.

Since $J^{2}=-1$, we may decompose $\left(T_{M} \oplus T_{M}^{*}\right) \otimes_{\mathbb{R}} \mathbb{C}$ into the $\pm i$ eigenvalue subspaces of $J$ :

$$
\left(T_{M} \oplus T_{M}^{*}\right) \otimes_{\mathbb{R}} \mathbb{C}=E+\bar{E},
$$

where $E$ be the $+i$-eigenvalue subspace.

If there is an $H \in H^{3}(M, \mathbb{R})$, we can define $H$-twisted Courant bracket as follow:

Let $A=X+\xi, B=Y+\eta \in\left(T_{M} \oplus T_{M}^{*}\right) \otimes_{\mathbb{R}} \mathbb{C}$, where $X, Y \in T_{M} \otimes_{\mathbb{R}}$ $\mathbb{C}, \xi, \eta \in T_{M}^{*} \otimes_{\mathbb{R}} \mathbb{C}$,

$$
\begin{aligned}
{[A, B]_{H} } & :=[X+\xi, Y+\eta]_{H} \\
& :=[X, Y]+L_{X} \eta-L_{Y} \xi-\frac{1}{2} d\left(\iota_{X} \eta-\iota_{Y} \xi\right)+\iota_{Y} \iota_{X} H
\end{aligned}
$$

where $[X, Y]:=X Y-Y X, L_{X} \eta:=d \iota_{X} \eta+\iota_{X} d \eta, \iota_{X}$ means the contraction by the vector field $X$.

If $J$ is an almost generalized complex structure, $[E, E]_{H} \subset E$,

$J$ is integrable, and $J$ is called the generalized complex structure. See Definition 4.36 in [2]. From Proposition 4.5 in [2], we have also known that the generalized complex manifold has even real dimension, so from now on, we assume that $\operatorname{dim}_{\mathbb{C}} M=n$.We have also known the the generalized complex manifold has even real dimension, so we assume that $\operatorname{dim}_{\mathbb{C}} M=n$ in the following.

This Courant bracket restricts to a Lie bracket on $E^{*} \cong \bar{E}$ with respect to the pair in Definition 2.1, and this Lie bracket can be extended into a Schouten bracket on $\wedge^{*} T_{M}^{*}$, which we continue to denote by $[\cdot, \cdot]_{H}$; it is defined by

$[A, B]_{H}:=\sum_{i, j}\left[A_{i}, B_{j}\right]_{H} \wedge A_{1} \wedge \cdots \wedge \hat{A}_{i} \wedge \cdots \wedge A_{p} \wedge B_{1} \wedge \cdots \wedge \hat{B}_{j} \wedge \cdots \wedge B_{q}$ where $A=A_{1} \wedge \cdots \wedge A_{p} \in \wedge^{p} E^{*}, \quad B=B_{1} \wedge \cdots \wedge B_{q} \in \wedge^{q} E^{*}, \quad \hat{A}_{i}$ means omit $A_{i}$. 
Specially, if $\epsilon \in \wedge^{2} E^{*},[\epsilon, \epsilon]_{H} \in \wedge^{3} E^{*}$ by definition.

Next, we define Clifford actions on $\wedge^{*} T_{M}^{*}$.

Definition 2.3. Let $\alpha \in \wedge^{*} T_{M}^{*}$ and $X+\xi \in\left(T_{M} \oplus T_{M}^{*}\right) \otimes_{\mathbb{R}} \mathbb{C}$, where $X \in$ $T_{M} \otimes_{\mathbb{R}} \mathbb{C}, \xi \in T_{M}^{*} \otimes_{\mathbb{R}} \mathbb{C}$, we can define Clifford actions on $\wedge^{*} T_{M}^{*}$ :

$$
(X+\xi) \alpha:=\iota_{X} \alpha+\xi \wedge \alpha
$$

Since $J$ is integrable, we have $<\bar{E}, \bar{E}>=0$, so

$$
A B \alpha+B A \alpha=0
$$

where $A, B \in E^{*}$. More generally,

$$
A B \alpha=(-1)^{p q} B A \alpha,
$$

where $A \in \wedge^{p} E^{*}, B \in \wedge^{q} E^{*}$.

Definition 2.4. In complex case, we have the Hodge decomposition. In generalized case, we can also define

$$
\begin{aligned}
& U_{0}:=\left\{\rho \in \wedge^{*} T_{M}^{*} \mid E \rho=0\right\}, \\
& U_{k}:=\wedge^{k} E^{*} \cdot U_{0} .
\end{aligned}
$$

One can show that $U_{0}$ is a complex line bundle in $\wedge^{*} T_{M}^{*}$. See Definition 20 in Page 34 [4]. we call it the canonical bundle of $J$. And $U_{k}$ is the $(n-k) i$ eigenvalue subspace of $J$.

Thus we can get the decomposition of $\wedge^{*} T_{M}^{*}$ :

$$
\wedge^{*} T_{M}^{*}=U_{0} \oplus U_{1} \oplus \ldots \oplus U_{2 n}
$$

where $n=\operatorname{dim}_{\mathbb{C}} M$. See P13 in [1].

Definition 2.5. Like the de Rham differential in complex case, we define the twisted de Rham differential $d_{H}$ on $\wedge^{*} T_{M}^{*}$ by

$$
\begin{aligned}
d_{H}: \wedge^{*} T_{M}^{*} & \rightarrow \wedge^{*} T_{M}^{*}, \\
\alpha & \mapsto d \alpha-H \wedge \alpha
\end{aligned}
$$

where $H \in H^{3}(M, \mathbb{R})$. 
Some Results of Deformations on Compact $H$-twisted Generalized...137

We can also define the twisted Dolbeault operator $\partial_{H}$ and $\bar{\partial}_{H}$ by

$$
\begin{aligned}
& \partial_{H}:=\pi_{k-1} \circ d_{H}: U_{k} \rightarrow U_{k-1}, \\
& \bar{\partial}_{H}:=\pi_{k+1} \circ d_{H}: U_{k} \rightarrow U_{k+1},
\end{aligned}
$$

where $\pi_{K}$ is the projection onto $U_{k}$.

Then $J$ is integrable if and only if $d_{H}=\partial_{H}+\bar{\partial}_{H}$. See P51 in [2].

Now, we define the compact generalized Kähler manifolds and the compact generalized Calabi-Yau manifold.

Definition 2.6. Let $(M, J)$ be a compact generalized complex manifold, if there exists another generalized complex structure $I$, such that $I J=J I$, then we call $(M, J, I)$ a compact $H$-twisted generalized manifold. And also exists a global nowhere zero $\rho_{0} \in U_{0}$, satisfying

$$
d_{H} \rho_{0}=0,
$$

we call $(M, J, I)$ a compact $H$-twisted generalized Calabi-Yau manifold, and $U_{0}$ is a trivial line bundle.

We still can define the inner product similar to the complex case which we call it as Born-Infeld inner products.

Definition 2.7. See P3 in [3]. Let $M$ be a compact H-twisted Generalized Kähler manifold, we can define a positive-definite metric on $\left(T_{M} \oplus T_{M}^{*}\right) \otimes_{\mathbb{R}}$ $\mathbb{C}$ by

$$
\begin{aligned}
\left(\left(T_{M} \oplus T_{M}^{*}\right) \otimes_{\mathbb{R}} \mathbb{C}\right) \otimes\left(\left(T_{M} \oplus T_{M}^{*}\right) \otimes_{\mathbb{R}} \mathbb{C}\right) & \rightarrow C^{\infty}(M), \\
A, B & \mapsto<G A, B>
\end{aligned}
$$

where $G A:=-I J A, \quad A \in\left(T_{M} \oplus T_{M}^{*}\right) \otimes_{\mathbb{R}} \mathbb{C}$. And one can show that $G^{2}=1$.

The restriction of this metric to the the sub-bundle $T_{M}$ is the Riemannian metric $g-b g^{-1} b$, where $g$ is a Riemannian metric and $b$ is a 2-form. And the volume element induced by this metric is 


$$
\begin{aligned}
\operatorname{vol}_{G} & =\sqrt{\operatorname{det}\left(g-b g^{-1} b\right)} \\
& =\frac{\operatorname{det}(g+b)}{\sqrt{\operatorname{detg}}} .
\end{aligned}
$$

Next, we define the $*$ operator by

$$
*=A_{1} A_{2} \ldots A_{n},
$$

which is a product of an oriented orthonormal basis for $C_{+}$, where $C_{+}$is the +1 -eigenvalue subspace of $G$ on $\left(T_{M} \oplus T_{M}^{*}\right) \otimes_{\mathbb{R}} \mathbb{C}$, since $G^{2}=1$. And one can also show that $C_{+}$is a real space, $A_{i}=\bar{A}_{i}$. So $*$ is a real operator, i.e. $*=\bar{*}$.

Then we can get

$$
\begin{aligned}
*^{2} & :=A_{1} A_{2} \ldots A_{n} A_{1} A_{2} \ldots A_{n} \\
& =(-1)^{n(n-1) / 2} A_{n} A_{n-1} \ldots A_{1} A_{1} A_{2} \ldots A_{n} \\
& =(-1)^{n(n-1) / 2}
\end{aligned}
$$

where $n=\operatorname{dim}_{\mathbb{C}} M$. The 2nd equality holds since $A_{i} A_{j}=-A_{j} A_{i}$ and, the 3rd equality holds since $A_{i}$ be orthonormal with respect to the pair $\langle\cdot, \cdot\rangle$ definite in Definition 2.1.

We now define the positive-definite Hermitian inner product on $\Gamma\left(M, \wedge^{*} T_{M}^{*}\right)$ which we call the Born-Infeld inner product by

$$
\begin{aligned}
(\alpha, \beta) & :=\int_{M}<\alpha, \sigma(*) \bar{\beta}> \\
\|\alpha\|^{2} & :=(\alpha, \alpha)
\end{aligned}
$$

where $\langle\alpha, \sigma(*) \bar{\beta}>$ is definite by

$$
\begin{aligned}
<\alpha, \sigma(*) \bar{\beta}> & =G(\alpha, \beta)<1, \sigma(*) 1> \\
& =G(\alpha, \beta) \text { vol }_{G} \\
& =g\left(e^{-b} \alpha, e^{-b} \beta\right) \text { vol }_{g}
\end{aligned}
$$

$G(\alpha, \beta)$ is a positive-definite metric on $\wedge^{*} T_{M}^{*}$ satisfying $G(1,1)=1$, $\sigma\left(A_{1} \ldots A_{n}\right):=A_{n} \ldots A_{1}=(-1)^{n(n-1) / 2} A_{1} \ldots A_{n}$, and $g$ is the Riemannian metric $b$ is some 2 -form definite above. 
Some Results of Deformations on Compact $H$-twisted Generalized...139

Remark 2.8. If $M$ is a Kähler manifold, we get that $b=0, *$ is just the ordinary Hodge *-operator and $(\cdot, \cdot)$ is the ordinary positive-definite inner product on differential forms definite in [15].

Lemma $2.9\left(\partial_{H} \bar{\partial}_{H}\right.$-Lemma). We say $(M, J)$ satisfies the $\partial_{H} \bar{\partial}_{H}$-Lemma if

$$
\operatorname{Im}\left(\partial_{H}\right) \cap \operatorname{Ker}\left(\bar{\partial}_{H}\right)=\operatorname{Im}\left(\bar{\partial}_{H}\right) \cap \operatorname{Ker}\left(\partial_{H}\right)=\operatorname{Im}\left(\partial_{H} \bar{\partial}_{H}\right) .
$$

One can show that a compact $H$-twisted generalized Kähler manifold $(M, J)$ satisfies the $\partial_{H} \bar{\partial}_{H}$-Lemma. See Corollary 4.2 in Page 6 [3].

From now on, we only discuss about the compact $H$-twisted generalized Kähler manifold $(M, J)$ which satisfies the $\partial_{H} \bar{\partial}_{H}$-Lemma.

We also have some elliptic operator similar to the complex case.

Definition 2.10. We now let $\partial_{H}^{*}, \bar{\partial}_{H}^{*}, d_{H}^{*}$ be the dual operators of $\partial_{H}, \bar{\partial}_{H}, d_{H}$ with respect to the inner product $(\cdot, \cdot)$ respectively, i.e.

$$
\begin{aligned}
& \left(\partial_{H} \alpha, \beta\right)=\left(\alpha, \partial_{H}^{*} \beta\right), \\
& \left(\bar{\partial}_{H} \alpha, \beta\right)=\left(\alpha, \bar{\partial}_{H}^{*} \beta\right), \\
& \left(d_{H} \alpha, \beta\right)=\left(\alpha, d_{H}^{*} \beta\right) .
\end{aligned}
$$

And we also define the Laplacian operators by

$$
\begin{aligned}
\triangle_{d_{H}} & :=d_{H} d_{H}^{*}+d_{H}^{*} d_{H}, \\
\triangle_{\partial_{H}} & :=\partial_{H} \partial_{H}^{*}+\partial_{H}^{*} \partial_{H}, \\
\triangle_{\bar{\partial}_{H}} & :=\bar{\partial}_{H} \bar{\partial}_{H}^{*}+\bar{\partial}_{H}^{*} \bar{\partial}_{H} .
\end{aligned}
$$

Then one can show that $\triangle_{d_{H}}, \triangle_{\partial_{H}}, \triangle_{\bar{\partial}_{H}}$ are self-duality operators with respect to inner product $(\cdot, \cdot)$ and

$$
\begin{aligned}
\triangle_{d_{H}} & =2 \triangle_{\partial_{H}}=2 \triangle_{\bar{\partial}_{H}}, \\
i d & =\mathbb{H}+G \triangle_{\bar{\partial}_{H}}=\mathbb{H}+\triangle_{\bar{\partial}_{H}} G,
\end{aligned}
$$

where $\mathbb{H}$ is the projection onto $H_{\bar{\partial}_{H}}\left(M, \wedge^{*} T_{M}^{*}\right)$, and $G$ is the Green operator corresponding to $\triangle_{\bar{\partial}_{H}}$. See P6 in [3].

We can also get the Hodge decomposition in twisted cohomology:

$$
\begin{array}{r}
H_{d_{H}}^{\text {even } / \text { odd }}\left(M, \wedge^{*} T_{M}^{*}\right)=H_{\bar{\partial}_{H}}^{0}\left(M, \wedge^{*} T_{M}^{*}\right) \oplus H_{\bar{\partial}_{H}}^{2}\left(M, \wedge^{*} T_{M}^{*}\right) \oplus \\
\cdots \oplus H_{\bar{\partial}_{H}}^{2 n}\left(M, \wedge^{*} T_{M}^{*}\right) .
\end{array}
$$




\section{Some lemmas}

In this section, we review some basic propositions of compact $H$-twisted generalized Calabi-Yau manifolds which will be used in this paper.

Lemma 3.1. The complex $\left(\wedge^{*} T_{M}^{*}, \bar{\partial}_{H}\right)$ is definite in Definition 2.3. We now introduce the complex $\left(\wedge^{p} E^{*}, d_{E}\right)$ :

$d_{E}: \wedge^{k} E^{*} \rightarrow \wedge^{k+1} E^{*}$ is the Lie derivation define by

$$
\begin{aligned}
d_{E} A\left(X_{0}, \ldots X_{k}\right):= & \sum_{i}(-1)^{i} a\left(X_{i}\right) A\left(X_{0}, \ldots, \hat{X}_{i}, \ldots, X_{k}\right) \\
& +\sum_{i<j}(-1)^{i+j} A\left(\left[X_{i}, X_{j}\right]_{H}, X_{0}, \ldots, \hat{X}_{i}, \ldots, \hat{X}_{j}, \ldots, X_{k}\right),
\end{aligned}
$$

where $A \in \wedge^{k} E^{*}, X_{i} \in E, a: E \rightarrow T_{M}$ is the projection which is called the anchor.

On compact generalized Kähler manifold $M$, we have

$$
\bar{\partial}_{H}(A \rho)=\left(d_{E} A\right) \bar{\rho}+(-1)^{p} A \bar{\partial}_{H} \rho,
$$

where $A \in \wedge^{p} E^{*}, \rho \in \wedge^{*} T_{M}^{*}$.

So, on compact generalized Calabi-Yau manifold $M$, we have the relationship between two complex $\left(\wedge^{*} T_{M}^{*}, \bar{\partial}_{H}\right)$ and $\left(\wedge^{p} E^{*}, d_{E}\right)$. See P8 in [14], or [2].

Then we have the two complex are isomorphism:

$$
\begin{gathered}
\left(\wedge^{p} E^{*}, d_{E}\right) \cong\left(\wedge^{*} T_{M}^{*}, \bar{\partial}_{H}\right), \\
\left(d_{E} A\right) \rho_{0}=\bar{\partial}_{H}\left(A \rho_{0}\right),
\end{gathered}
$$

where $A \in \wedge^{p} E^{*}, \rho_{0} \in U_{0}$ is the global nowhere zero $d_{H}$-closed section which is fixed and we call it the pure spinor for $J$.

Next, we have the following Clifford actions on compact generalized Kähler manifold $M$. See [13, 14].

Lemma 3.2. Let $\alpha \in \wedge^{*} T_{M}^{*}$ be a smooth differential form, $A \in \wedge^{p} E^{*}, B \in$ $\wedge^{q} E^{*}$, we have 
Some Results of Deformations on Compact $H$-twisted Generalized...141

$$
\begin{aligned}
d_{H}(A B \alpha)= & (-1)^{p} A d_{H}(B \alpha)+(-1)^{(p-1) q} B d_{H}(A \alpha)+(-1)^{p-1}[A, B]_{H} \alpha \\
& +(-1)^{p+q+1} A B d_{H} \alpha, \\
\partial_{H}(A B \alpha)= & (-1)^{p} A \partial_{H}(B \alpha)+(-1)^{(p-1) q} B \partial_{H}(A \alpha)+(-1)^{p-1}[A, B]_{H} \alpha \\
& +(-1)^{p+q+1} A B \partial_{H} \alpha, \\
\bar{\partial}_{H}(A B \alpha)= & (-1)^{p} A \bar{\partial}_{H}(B \alpha)+(-1)^{(p-1) q} B \bar{\partial}_{H}(A \alpha) \\
& +(-1)^{p+q+1} A B \bar{\partial}_{H} \alpha .
\end{aligned}
$$

Further more, if $d_{H} \rho_{0}=\partial_{H} \rho_{0}=\bar{\partial}_{H} \rho_{0}=0$ and $\partial_{H}\left(A \rho_{0}\right)=\partial_{H}\left(B \rho_{0}\right)=$ $0, A, B \in \wedge^{2} E^{*}$, we have

$$
\partial_{H}\left(A B \rho_{0}\right)=-[A, B]_{H} \rho_{0} .
$$

The following inequivalent we will need to prove the convergence.

Lemma 3.3. On compact generalized Kähler manifold $M$. Let $s \geq 2 \in \mathbb{N}$, $\alpha \in \mathbb{R}, 0<\alpha<1$ is fixed. Then there exist positive constants $c_{1}, c_{2}, c_{3}$ which only depend on $s, \alpha, H$, and the manifold $M$ itself, such that for any $A, B \in$ $\wedge^{*} E^{*}$,

$$
\begin{aligned}
\left\|[A, B]_{H}\right\|_{s+\alpha} & \leq c_{1}\|A\|_{s+1+\alpha} \cdot\|B\|_{s+1+\alpha} \\
\left\|d_{E}^{*} A\right\|_{s+\alpha} & \leq c_{2}\|B\|_{s+1+\alpha} \\
\left\|G_{d_{E}} A\right\|_{s+\alpha} & \leq c_{3}\|A\|_{s-2+\alpha}
\end{aligned}
$$

where $G_{d_{E}}$ is the Green operator corresponding to $\triangle_{d_{E}}$, and $\|\cdot\|_{s+\alpha}$ means the Hölder norms definite in [11] and we consider the norms of $A \in$ $\wedge^{p} E$ the same as the norms of $A \rho_{0} \in \wedge^{*} T_{M}^{*}$ since Lemma 3.1.

So we have

$$
\begin{aligned}
\left\|d_{E}^{*} G_{d_{E}}[A, B]_{H}\right\|_{s+\alpha} & \leq c_{1} c_{2} c_{3}\|A\|_{s+\alpha}\|B\|_{s+\alpha} \\
& :=(2 C)\|A\|_{s+\alpha}\|B\|_{s+\alpha} .
\end{aligned}
$$

On compact generalized Calabi-Yau manifold $M$. If $\partial_{H}\left(A \rho_{0}\right)=$ $\partial_{H}\left(B \rho_{0}\right)=0$, we have 


$$
\left\|\bar{\partial}_{H}^{*} G \partial_{H}\left(A B \rho_{0}\right)\right\|_{s+\alpha} \leq(2 c)\left\|A \rho_{0}\right\|_{s+\alpha}\left\|B \rho_{0}\right\|_{s+\alpha}
$$

by using the formula 3.1$)$ in Lemma 3.2 .

Proof. (1) Locally, $\left.A\right|_{U}=A_{J}^{I} \frac{\partial}{\partial x^{I}} \wedge d x^{J}$. Then $\|A\|_{s+\alpha}^{U}:=\left\|A_{J}^{I}\right\|_{s+\alpha}$ just as the definition in Calabi-Yau case.

By the definition of the $d_{E}$, it is sufficient to prove the inequality holds for $A=X+\varphi, B=Y+\eta \in E^{*}$. Since by direct computation, we have

$$
\begin{aligned}
& \iota_{Y_{0}} d \eta_{1}= \iota_{Y_{0}^{i} \frac{\partial}{\partial x^{i}}} \frac{\partial \eta_{1 j}}{\partial x^{p}} d x^{p} \wedge d x^{j} \\
&= \iota_{Y_{0}^{i} \frac{\partial}{\partial x^{i}}} \frac{1}{2}\left(\frac{\partial \eta_{1 j}}{\partial x^{p}}-\frac{\partial \eta_{1 p}}{\partial x^{j}}\right) d x^{p} \wedge d x^{j} \\
&= \frac{1}{2}\left(\frac{\partial \eta_{1 j}}{\partial x^{p}}-\frac{\partial \eta_{1 p}}{\partial x^{j}}\right) Y_{0}^{p} d x^{j}-\frac{1}{2}\left(\frac{\partial \eta_{1 j}}{\partial x^{p}}-\frac{\partial \eta_{1 p}}{\partial x^{j}}\right) Y_{0}^{j} d x^{p} \\
&= \frac{1}{2}\left(\frac{\partial \eta_{1 p}}{\partial x^{j}}-\frac{\partial \eta_{1 j}}{\partial x^{p}}\right) Y_{0}^{j} d x^{p}-\frac{1}{2}\left(\frac{\partial \eta_{1 j}}{\partial x^{p}}-\frac{\partial \eta_{1 p}}{\partial x^{j}}\right) Y_{0}^{j} d x^{p} \\
&=\left(\frac{\partial \eta_{1 p}}{\partial x^{j}}-\frac{\partial \eta_{1 j}}{\partial x^{p}}\right) Y_{0}^{j} d x^{p}, \\
& \frac{1}{2} d \circ \iota_{Y_{0}} \eta_{1}=\frac{1}{2} d\left(Y_{0}^{i} \eta_{1 i}\right) \\
& \quad=\frac{1}{2}\left(\frac{\partial Y_{0}^{i}}{\partial x^{p}} \eta_{1 i}+Y_{0}^{i} \frac{\partial \eta_{1 i}}{\partial x^{p}}\right) d x^{p} \\
& \iota_{Y_{1} \iota_{Y_{0}} H}= \\
&=\iota_{Y_{1}} \iota_{Y_{0}}\left(\frac{1}{3 !} H_{i j k} d x^{i} \wedge d x^{j} \wedge d x^{k}\right) \\
&=\iota_{Y_{1}}\left(\frac{1}{3 !} Y_{0}^{p}\left(H_{p i j}-H_{i p j}+H_{i j p}\right) d x^{i} \wedge d x^{j}\right) \\
&=\iota_{Y_{1}}\left(\frac{1}{2 !} Y_{0}^{p} H_{i j p} d x^{i} \wedge d x^{j}\right) \\
&=\frac{1}{2 !} Y_{0}^{p} Y_{1}^{q}\left(H_{q j p}-H_{j q p}\right) d x^{j} \\
&=Y_{0}^{p} Y_{1}^{q} H_{q j p} d x^{j} \\
& Y_{0}^{p} Y_{1}^{q} H_{p q j} d x^{j},
\end{aligned}
$$


Some Results of Deformations on Compact $H$-twisted Generalized...143

$$
\begin{aligned}
{[A, B]_{H}:=} & {\left[Y_{0}+\eta_{0}, Y_{1}+\eta_{1}\right]_{H} } \\
:= & {\left[Y_{0}, Y_{1}\right]+L_{Y_{0}} \eta_{1}-L_{Y_{1}} \eta_{0}-\frac{1}{2} d\left(\iota_{Y_{0}} \eta_{1}-\iota_{Y_{1}} \eta_{0}\right)+\iota_{Y_{1}} \iota_{Y_{0}} H } \\
= & {\left[Y_{0}^{i} \frac{\partial}{\partial x^{i}}, Y_{1}^{j} \frac{\partial}{\partial x^{j}}\right]+d \circ \iota_{Y_{0}} \eta_{1}+\iota_{Y_{0}} d \eta_{1}-\frac{1}{2} d \iota_{Y_{0}} \eta_{1} } \\
& -d \circ \iota_{Y_{1}} \eta_{0}-\iota_{Y_{1}} d \eta_{0}+\frac{1}{2} d \iota_{Y_{1}} \eta_{0}+\iota_{Y_{1}} \iota_{Y_{0}} H \\
= & {\left[Y_{0}^{i} \frac{\partial}{\partial x^{i}}, Y_{1}^{j} \frac{\partial}{\partial x^{j}}\right]+\frac{1}{2} d \circ \iota_{Y_{0}} \eta_{1}+\iota_{Y_{0}} d \eta_{1} } \\
& -\frac{1}{2} d \circ \iota_{Y_{1}} \eta_{0}-\iota_{Y_{1}} d \eta_{0}+\iota_{Y_{1}} \iota_{Y_{0}} H \\
= & \left(Y_{0}^{i} \frac{\partial Y_{1}^{j}}{\partial x^{i}}-Y_{1}^{i} \frac{\partial Y_{0}^{j}}{\partial x^{i}}\right) \frac{\partial}{\partial x^{j}}+\frac{1}{2}\left(\frac{\partial Y_{0}^{i}}{\partial x^{p}} \eta_{1 i}+Y_{0}^{i} \frac{\partial \eta_{1 i}}{\partial x^{p}}\right. \\
& \left.-\frac{\partial Y_{1}^{i}}{\partial x^{p}} \eta_{10}-Y_{1}^{i} \frac{\partial \eta_{0 i}}{\partial x^{p}}\right) d x^{p}+\left(\frac{\partial \eta_{1 p}}{\partial x^{j}} Y_{0}^{j}-\frac{\partial \eta_{1 j}}{\partial x^{p}} Y_{0}^{j}\right. \\
& \left.-\frac{\partial \eta_{0 p}}{\partial x^{j}} Y_{1}^{j}+\frac{\partial \eta_{0 j}}{\partial x^{p}} Y_{1}^{j}\right) d x^{p}+Y_{0}^{i} Y_{1}^{j} H_{i j p} d x^{p} \\
= & \left(Y_{0}^{i} \frac{\partial Y_{1}^{j}}{\partial x^{i}}-Y_{1}^{i} \frac{\partial Y_{0}^{j}}{\partial x^{i}}\right) \frac{\partial}{\partial x^{j}} \\
& +\left(\frac{1}{2}\left(\frac{\partial Y_{0}^{i}}{\partial x^{p}} \eta_{1 i}-\frac{\partial Y_{1}^{i}}{\partial x^{p}} \eta_{0 i}\right)+\left(\frac{\partial \eta_{1 p}}{\partial x^{j}} Y_{0 j}-\frac{\partial \eta_{0 p}}{\partial x^{j}} Y_{1 j}\right)\right. \\
& \left.+\frac{1}{2}\left(Y_{1}^{i} \frac{\partial \eta_{0 i}}{\partial x^{p}}-Y_{0}^{i} \frac{\partial \eta_{1 i}}{\partial x^{p}}\right)+Y_{0}^{i} Y_{1}^{j} H_{i j p}\right) d x^{p}, \\
&
\end{aligned}
$$

where $x^{i}$ can be $z^{i}$ or $\overline{z^{i}}, H=\frac{1}{3 !} H_{i j k} d x^{i} \wedge d x^{j} \wedge d x^{k}, H_{i j k}$ is skew-symmetric with respect to $i, j, k$.

We also have known that $H$ is fixed, $M$ is compact, we can get some proper larger constant $c_{1}$ such that $c_{1} \geq \max \left\{H_{i j k}, \frac{\partial^{\gamma} H_{i j k}}{\partial x^{\gamma}}:|\gamma| \leq k\right\}$. So by the definition of Hölder norms, we get the 1st inequality. 
(2) By the definition of the $d_{E}$, it is sufficient to prove the inequality holds for $A=X+\varphi \in E^{*}$. Since by direct computation, we have

$$
\begin{aligned}
& \left(d_{E}(X+\varphi)\right)\left(Y_{0}+\eta_{0}, Y_{1}+\eta_{1}\right) \\
:=\quad & \left(d_{E}\left(X^{i} \frac{\partial}{\partial x^{i}}+\varphi_{j} d x^{j}\right)\right)\left(Y_{0}^{k} \frac{\partial}{\partial x^{k}}+\eta_{0 l} d x^{l}, Y_{1}^{k} \frac{\partial}{\partial x^{k}}+\eta_{1 l} d x^{l}\right) \\
:=\quad & Y_{0}<X+Y_{1}+\eta_{1}>-Y_{1}<X+\varphi, Y_{0}+\eta_{0}> \\
& -<X+\varphi,\left[Y_{0}+\eta_{0}, Y_{1}+\eta_{1}\right]_{H}> \\
= & \frac{1}{2} Y_{0}^{i} \frac{\partial}{\partial x^{i}}\left(\varphi_{j} Y_{1}^{j}+X^{k} \eta_{1 k}\right)-\frac{1}{2} Y_{1}^{i} \frac{\partial}{\partial x^{i}}\left(\varphi_{j} Y_{0}^{j}+X^{k} \eta_{0 k}\right) \\
& <X^{i} \frac{\partial}{\partial x^{i}}+\varphi_{j} d x^{j},\left(Y_{0}^{i} \frac{\partial Y_{1}^{j}}{\partial x^{i}}-Y_{1}^{i} \frac{\partial Y_{0}^{j}}{\partial x^{i}}\right) \frac{\partial}{\partial x^{j}} \\
& +\left(\frac{1}{2}\left(\frac{\partial Y_{0}^{i}}{\partial x^{p}} \eta_{1 i}-\frac{\partial Y_{1}^{i}}{\partial x^{p}} \eta_{0 i}\right)+\left(\frac{\partial \eta_{1 p}}{\partial x^{j}} Y_{0 j}-\frac{\partial \eta_{0 p}}{\partial x^{j}} Y_{1 j}\right)\right. \\
& \left.+\frac{1}{2}\left(Y_{1}^{i} \frac{\partial \eta_{0 i}}{\partial x^{p}}-Y_{0}^{i} \frac{\partial \eta_{1 i}}{\partial x^{p}}\right)+Y_{0}^{i} Y_{1}^{j} H_{i j p}\right) d x^{p},> \\
= & \frac{1}{2}\left(Y_{0}^{i} Y_{1}^{j} \frac{\partial \varphi_{j}}{\partial x^{i}}-Y_{1}^{i} Y_{0}^{j} \frac{\partial \varphi_{j}}{\partial x^{i}}+Y_{0}^{i} \varphi_{j} \frac{\partial Y_{1}^{j}}{\partial x^{i}}-Y_{1}^{i} \varphi_{j} \frac{\partial Y_{0}^{j}}{\partial x^{i}}\right. \\
& \left.+Y_{0}^{i} \eta_{1 k} \frac{\partial X^{k}}{\partial x^{i}}-Y_{1}^{i} \eta_{0 k} \frac{\partial X^{k}}{\partial x^{i}}+X^{k} Y_{0}^{i} \frac{\partial \eta_{1 k}}{\partial x^{i}}-X^{k} Y_{1}^{i} \frac{\partial \eta_{0 k}}{\partial x^{i}}\right) \\
& +\frac{1}{2}\left(Y_{1}^{i} \varphi_{j} \frac{\partial Y_{0}^{j}}{\partial x^{i}}-Y_{0}^{i} \varphi_{j} \frac{\partial Y_{1}^{j}}{\partial x^{i}}\right) \\
& +\frac{1}{2}\left(X^{p} Y_{1}^{j} \frac{\partial \eta_{0 p}}{\partial x^{j}}-X^{p} Y_{0}^{j} \frac{\partial \eta_{1 p}}{\partial x^{j}}\right)+\frac{1}{4}\left(X^{p} \eta_{0 i} \frac{\partial Y_{1}^{i}}{\partial x^{p}}-X^{p} \eta_{1 i} \frac{\partial Y_{0}^{i}}{\partial x^{p}}\right) \\
& +\frac{1}{4}\left(X^{p} Y_{0}^{i} \frac{\partial \eta_{1 i}}{\partial x^{p}}-X^{p} Y_{1}^{i} \frac{\partial \eta_{0 i}}{\partial x^{p}}\right)-\frac{1}{2} X^{p} Y_{0}^{i} Y_{1}^{j} H_{i j p} \\
= & \frac{1}{2} Y_{0}^{i} Y_{1}^{j}\left(\frac{\partial \varphi_{j}}{\partial x^{i}}-\frac{\partial \varphi_{i}}{\partial x^{j}}\right)+\frac{1}{2}\left(Y_{0}^{i} \eta_{1 k} \frac{\partial X^{k}}{\partial x^{i}}-Y_{1}^{i} \eta_{0 k} \frac{\partial X^{k}}{\partial x^{i}}\right) \\
+ & \frac{1}{4} X^{p}\left(\eta_{0 i} \frac{\partial Y_{1}^{i}}{\partial x^{p}}-\eta_{1 i} \frac{\partial Y_{0}^{i}}{\partial x^{p}}\right)+\frac{1}{4} X^{p}\left(Y_{0}^{i} \frac{\partial \eta_{1 i}}{\partial x^{p}}-Y_{1}^{i} \frac{\partial \eta_{0 i}}{\partial x^{p}}\right) \\
& -\frac{1}{2} X^{p} Y_{0}^{i} Y_{1}^{j} H_{i j p}, \\
= &
\end{aligned}
$$

where $Y_{0}+\eta_{0}, Y_{1}+\eta_{1} \in E$. 
Some Results of Deformations on Compact $H$-twisted Generalized...145

So we can get that

$$
\begin{aligned}
d_{E}(X+\varphi):= & \frac{1}{2}\left(\left(\frac{\partial \varphi_{j}}{\partial x^{i}}-\frac{1}{2} X^{p} H_{i j p}\right) d x^{i} \wedge d x^{j}+\frac{1}{2} \frac{\partial X^{k}}{\partial x^{i}} d x^{i} \wedge \frac{\partial}{\partial x^{k}}\right. \\
& \left.+\frac{1}{2} \frac{\partial}{\partial x^{i}} \wedge X^{p} \frac{\partial}{\partial x^{p}} d x^{i}+\frac{1}{2} d x^{i} \wedge X^{p} \frac{\partial}{\partial x^{p}} \frac{\partial}{\partial x^{i}}\right) \\
:= & \frac{1}{2}\left(\left(\frac{\partial \varphi_{j}}{\partial x^{i}}-\frac{1}{2} X^{p} H_{i j p}\right) d x^{i} \wedge d x^{j}+d X^{k} \wedge \frac{\partial}{\partial x^{k}}\right. \\
& \left.\frac{1}{2} \frac{\partial}{\partial x^{i}} \wedge X d x^{i}+\frac{1}{2} d x^{i} \wedge X \frac{\partial}{\partial x^{i}}\right)
\end{aligned}
$$

Thus $d_{E}$ is an operator of order 1 .

In the above computation, we just assume that $A=X+\varphi \in E^{*} \subset$ $T_{M} \oplus T_{M}^{*}$, and we didn't use the condition $[E, E]_{H} \subset E$. There is another way to show $d_{E}$ is an operator of order 1 . If we choose a basis $E:\left\{e_{i}=\right.$ $\left.Y_{i}+\eta_{i}\right\}_{i=1}^{2 n}, E^{*}:\left\{e^{i}=X^{i}+\varphi^{i}\right\}_{i=1}^{2 n},\left[e_{j}, e_{k}\right]_{H}:=c_{j k}^{p} e_{p}, c_{j k}^{p} \in C^{\infty}(U)$. Also we let $f_{i} \in C^{\infty}(U)$.

Then we have

$$
\begin{aligned}
\left(d_{E}\left(f_{i} e^{i}\right)\right)\left(e_{j}, e_{k}\right):= & a\left(e_{j}\right)<f_{i} e^{i}, e_{k}>-a\left(e_{k}\right)<f_{i} e^{i}, e_{j}> \\
& -<f_{i} e^{i},\left[e_{j}, e_{k}\right]_{H}> \\
= & Y_{j}\left(f_{k}\right)-Y_{k}\left(f_{j}\right)-<f_{i} e^{i}, c_{j k}^{p} e_{p}> \\
= & Y_{j}^{p} \frac{\partial f_{k}}{\partial x^{p}}-Y_{k}^{p} \frac{\partial f_{j}}{\partial x^{p}}-f_{p} c_{j k}^{p} .
\end{aligned}
$$

Thus, we get

$$
d_{E}\left(f_{i} e^{i}\right)=\left(Y_{j}^{p} \frac{\partial f_{k}}{\partial x^{p}}-Y_{k}^{p} \frac{\partial f_{j}}{\partial x^{p}}-f_{p} c_{j k}^{p}\right) e^{j} \wedge e^{k} .
$$

Thus $d_{E}$ is an operator of order 1 since the coefficients only has $f, d f$ 's terms and the change of the basis not change the order of $d_{E}$.

We have also known that

$$
\begin{aligned}
d_{E}^{*}:= & * d_{E} *^{-1} \\
:= & A_{1} \cdots A_{n} \circ d_{E} \circ A_{n} \cdots A_{1} \\
= & \left(X_{1}+g\left(X_{1}\right)+b\left(X_{1}\right)\right) \cdots\left(X_{n}+g\left(X_{n}\right)+b\left(X_{n}\right)\right) \\
& \circ d_{E} \circ\left(X_{n}+g\left(X_{n}\right)+b\left(X_{n}\right)\right) \cdots\left(X_{1}+g\left(X_{1}\right)+b\left(X_{1}\right)\right) .
\end{aligned}
$$


The 3rd equality holds since every element in $C_{+}$can be represented as $X+g(X)+b(X)$ for some $X \in T_{M} \times_{\mathbb{R}} \mathbb{C}$, where $g, b$ is definite in Definition 2.7. See [8].

So we can get that $d_{E}^{*}$ is also an operator of order 1 . Since every $X_{i}, g, b$ is fixed, only depend on the manifold $M$ itself, and by the definition of the Hölder norms, we can get the 2nd inequality.

(3) Since $d_{E}$ and $d_{E}^{*}$ are both operators of order 1 , we get that $\triangle_{d_{E}}:=$ $d_{E} d_{E}^{*}+d_{E}^{*} d_{E}$ is an operator of order 2 just as the harmonic operator in Calabi-Yau case.

Since the Green operator $G_{d_{E}}$ is a strongly elliptic operator just as the Green operator in Calabi-Yau case, we can use the same proof as Lemma 5.7 at P276 and Theorem 4.3 in P436 in 11 just replace the vector bundle $\wedge^{*} T_{M}^{*}$ by $\wedge^{*} T_{M} \oplus \wedge^{*} T_{M}^{*}$ and replace the local representation $A=\frac{1}{3 !} A_{i j k} d x^{i} \wedge d x^{j} \wedge d x^{k}$ by $A=\frac{1}{3 !}\left(A_{i j k} d x^{i} \wedge d x^{j} \wedge d x^{k}+A_{j k}^{i} \frac{\partial}{\partial x^{i}} \wedge d x^{j} \wedge\right.$ $d x^{k}+A_{k}^{i j} \frac{\partial}{\partial x^{i}} \wedge \frac{\partial}{\partial x^{j}} \wedge d x^{k}+A^{i j k} \frac{\partial}{\partial x^{i}} \wedge \frac{\partial}{\partial x^{j}} \wedge \frac{\partial}{\partial x^{k}}$ to get the 3rd inequality.

The following convergent power series we will use to prove the convergence.

Lemma 3.4 (See Lemma 4.1 in [12]). Let $x_{1}=a$ be a constant, and $x_{k}=c \sum_{i=1}^{k-1} x_{i} x_{k-i}$, where $c$ is a constant, then $\sum_{i=1}^{\infty} x_{i} t^{i}$ converge on $|t| \leq$ $\frac{1}{4 a c}$.

\section{A generalized version of $\partial_{H} \bar{\partial}_{H^{-}}$lemma}

In this section, we will prove a generalized version of $\partial_{H} \bar{\partial}_{H}$-lemma on a compact $H$-twisted generalized Kähler manifold which is similar to the result in Kähler case in [12. This lemma will be used in the next section to construct the deformations.

Lemma 4.1. For any $\rho \in \wedge^{*} T_{M}^{*}$,

$$
\begin{aligned}
\left\|\bar{\partial}_{H}^{*} G \rho\right\|^{2} & \leq(\rho, G \rho), \\
\left\|\bar{\partial}_{H}^{*} G \partial_{H} \rho\right\|^{2} & \leq\|\rho\|^{2} .
\end{aligned}
$$


Some Results of Deformations on Compact $H$-twisted Generalized...147

Proof. (1)

$$
\begin{aligned}
\left\|\bar{\partial}_{H}^{*} G \rho\right\|^{2} & :=\left(\bar{\partial}_{H}^{*} G \rho, \bar{\partial}_{H}^{*} G \rho\right) \\
& =\left(\bar{\partial}_{H} \bar{\partial}_{H}^{*} G \rho, G \rho\right) \\
& =\left(\rho-\mathbb{H} \rho-\bar{\partial}_{H}^{*} \bar{\partial}_{H} G \rho, G \rho\right) \\
& =(\rho, G \rho)-\left(\bar{\partial}_{H}^{*} \bar{\partial}_{H} G \rho, G \rho\right) \\
& =(\rho, G \rho)-\left(\bar{\partial}_{H} G \rho, \bar{\partial}_{H} G \rho\right) \\
& \leq(\rho, G \rho)
\end{aligned}
$$

The 4th equality holds since $\left.\mathbb{H} \rho \in H_{\bar{\partial}_{H}}^{*}(M, S)\right) \perp G \rho \in \operatorname{Im} \triangle_{\bar{\partial}_{H}}$ with respect to the inner product $(\cdot, \cdot)$ which is definite in Definition 2.7. And the equality holds if and only if $\bar{\partial}_{H} G \rho=0$.

$(2)$

$$
\begin{aligned}
\left\|\bar{\partial}_{H}^{*} G \partial_{H} \rho\right\|^{2} & :=\left(\bar{\partial}_{H}^{*} G \partial \rho, \bar{\partial}_{H}^{*} G \partial_{H} \rho\right) \\
& =\left(\bar{\partial}_{H} \bar{\partial}_{H}^{*} G \partial_{H} \rho, G \partial_{H} \rho\right) \\
& =\left(\triangle_{\bar{\partial}_{H}} G \partial_{H} \rho, G \partial_{H} \rho\right)-\left(\bar{\partial}_{H}^{*} G \bar{\partial}_{H} \partial \rho, G \partial_{H} \rho\right) \\
& =\left(\rho, \partial_{H}^{*} G \partial_{H} \rho\right)-\left(G \bar{\partial}_{H} \partial_{H} \rho, G \bar{\partial}_{H} \partial_{H} \rho\right) \\
& =\left(\rho, \triangle_{\bar{\partial}_{H}} G \rho\right)-\left(\rho, \partial_{H} \partial_{H}^{*} G \rho\right)-\left\|G \bar{\partial}_{H} \partial_{H} \rho\right\|^{2} \\
& =\left(\rho, \rho-\mathbb{H} \rho-\partial_{H} \partial_{H}^{*} G \rho\right)-\left\|G \bar{\partial}_{H} \partial_{H} \rho\right\|^{2} \\
& =\|\rho\|^{2}-\|\mathbb{H} \rho\|^{2}-\left(\partial_{H}^{*} \rho, G \partial_{H}^{*} \rho\right)-\left\|G \bar{\partial}_{H} \partial_{H} \rho\right\|^{2} \\
& \leq\|\rho\|^{2}
\end{aligned}
$$

The 3rd and 4th equality holds since $\triangle_{\partial_{H}}=\triangle_{\bar{\partial}_{H}}$, and $\bar{\partial}_{H} G=G \bar{\partial}_{H}$ which we give a simple proof: let $G \bar{\partial}_{H} \rho:=\beta \in \operatorname{Im} \Delta_{\bar{\partial}_{H}}, G \rho:=\alpha \in \operatorname{Im} \Delta_{\bar{\partial}_{H}}$, then $\Delta_{\bar{\partial}_{H}} \alpha=\rho-\mathbb{H} \rho, \bar{\partial}_{H} \rho=\Delta_{\bar{\partial}_{H}} \beta$. since $\bar{\partial}_{H}^{*} \bar{\partial}_{H}^{2} \alpha+\bar{\partial}_{H} \bar{\partial}_{H}^{*} \bar{\partial}_{H} \alpha=\bar{\partial}_{H} \bar{\partial}_{H}^{*} \bar{\partial}_{H} \alpha+$ $\bar{\partial}_{H}^{2} \bar{\partial}_{H}^{*} \alpha$, we have that $\Delta_{\bar{\partial}_{H}} \bar{\partial}_{H} \alpha=\bar{\partial}_{H} \Delta_{\bar{\partial}_{H}} \alpha=\bar{\partial}_{H} \rho-\bar{\partial}_{H}(\mathbb{H} \rho)=\bar{\partial}_{H} \rho$. So $G \bar{\partial}_{H} \rho=G \Delta_{\bar{\partial}_{H}} \bar{\partial}_{H} \alpha=\bar{\partial}_{H} \alpha=\bar{\partial}_{H} G \rho$. (Or see P147, theorem 5.2(d) in [21] and the elliptic proposition see Proposition 6 in Page 35 [4].). The last inequality holds since $\left.G_{\partial_{H}}\right|_{\operatorname{Im} \triangle_{\partial_{H}}}=\triangle_{\partial_{H}}^{-1}$, we assume $G_{\partial_{H}} \partial_{H}^{*} \rho:=\alpha$, then $\left(\partial_{H}^{*} \rho, G_{\partial_{H}} \partial_{H}^{*} \rho\right)=\left(\triangle_{\partial_{H}} \alpha, \alpha\right) \geq 0$, see more details in [7]. And the equality holds if and only if $\rho \in \operatorname{Im} \triangle_{\bar{\partial}_{H}}, \bar{\partial}_{H} \partial_{H} \rho=0$, and $\partial_{H}^{*} \rho=0$. 
Proposition 4.2. Let $(M, J)$ be a compact $H$-twisted Generalized CalabiYau manifold, then for any $\rho \in \wedge^{*} T_{M}^{*}$,

$$
s=\bar{\partial}_{H}^{*} G \partial_{H} \rho
$$

is a solution to the equation $\bar{\partial}_{H} s=\partial_{H} \rho$ with condition $\bar{\partial}_{H} \partial_{H} \rho=0$, such that

$$
\|s\|^{2} \leq\left(\partial_{H} \rho, G \partial_{H} \rho\right) .
$$

Moreover, if $\mathbb{H} s=0$, and $\bar{\partial}_{H}^{*} s=0, s$ is uniquely determined.

Proof.

$$
\begin{aligned}
\bar{\partial}_{H} s & =\bar{\partial}_{H} \bar{\partial}_{H}^{*} G \partial_{H} \rho \\
& =\partial_{H} \rho-\mathbb{H}_{H} \rho-\bar{\partial}_{H}^{*} \bar{\partial}_{H} G \partial_{H} \rho \\
& =\partial_{H} \rho-\bar{\partial}_{H}^{*} \bar{\partial}_{H} G \partial_{H} \rho \\
& =\partial_{H} \rho
\end{aligned}
$$

The 3rd equality holds since $\partial_{H} \rho \in \operatorname{Im} \partial_{H} \perp H_{\bar{\partial}_{H}}^{*}\left(M, \wedge^{*} T_{M}^{*}\right)$; the last equality holds since the assumption $\bar{\partial}_{H} \partial_{H} \rho=0$. So $s$ is a solution and

$$
\|s\|^{2} \leq\left(\partial_{H} \rho, G \partial_{H} \rho\right)
$$

holds by the Lemma 4.1 above.

We now prove the uniqueness of the solution under the assumptions given.

If there exists another solution $s^{\prime}$, we have $\bar{\partial}_{H}\left(s-s^{\prime}\right)=0$. Also we have $\mathbb{H}\left(s-s^{\prime}\right)=0, \bar{\partial}_{H}^{*}\left(s-s^{\prime}\right)=0$ by assumption, then $s-s^{\prime}=0$.

\section{An application on a compact $H$-twisted generalized Calabi-Yau manifold}

In [14], it has been proved that a sufficiently small deformations of a compact $H$-twisted generalized Calabi-Yau manifold are unobstructed. In this section, we shall re-state that the deformation can be $L^{2}$ convergence in a fixed neighbourhood by using another convergent power series which is parallel to that in [12]. 
Some Results of Deformations on Compact $H$-twisted Generalized...149

Theorem 5.1. Let $(M, J)$ be a compact $H$-twisted generalized Calabi-Yau manifold, then there exists a globally $L^{2}$ convergent power series which determines the deformations in $t<\frac{1}{4 a c}$,

$$
\epsilon(t) \rho_{0}=\sum_{i=1}^{N} \epsilon_{i} \rho_{0} t^{i}+\sum_{k \geq 2} \sum_{k_{1}+\cdots+k_{N}=k, k_{i} \geq 0} \epsilon_{k_{1} \cdots k_{N}} \rho_{0}\left(t^{1}\right)^{k_{1}} \cdots\left(t^{N}\right)^{k_{N}},
$$

which satisfies:

(1) $\bar{\partial}_{H} \epsilon(t) \rho_{0}+\frac{1}{2}[\epsilon(t), \epsilon(t)]_{H} \rho_{0}=0$;

(2) $\epsilon_{k_{1} \cdots k_{N}} \rho_{0}$ is $\bar{\partial}_{H}^{*}$-closed, and $\partial_{H}$-exact for any $k_{1}+\cdots+k_{N}=k \geq 2$;

(3) $\epsilon(t) \rho_{0}$ is $L^{2}$ convergence in $t<\frac{1}{4 a c}$.

Proof. In step 1, we recall the construction of the deformation $\epsilon(t)$, in step 2 , we prove that $\epsilon(t)$ is $L^{2}$ convergence in $t<\frac{1}{4 a c}$.

\section{Step 1:}

The construction of a power series solution $\epsilon(t)$ to the integrable condition has been given in [14], here we just recall it in order to make the proof more clearly.

The integrable condition which is called Maurer-Cartan equation is:

$$
d_{E} \epsilon(t)+\frac{1}{2}[\epsilon(t), \epsilon(t)]_{H}=0
$$

By Lemma 3.1, we can rewrite it:

$$
\bar{\partial}_{H}\left(\epsilon(t) \rho_{0}\right)+\frac{1}{2}[\epsilon(t), \epsilon(t)]_{H} \rho_{0}=0,
$$

where $\rho_{0} \in U_{0}$ is the canonical bundle.

Choose a basis of $H_{d_{E}}^{2}\left(M, \wedge^{*} E^{*}\right):\left\{\epsilon_{i}\right\}_{i=1}^{N}$. We now recall the construction of a power series solution $\epsilon(t)$ to the integrable condition in [14]. We write $\epsilon(t)$ in the form of

$$
\epsilon(t):=\epsilon_{i} t^{i}+\epsilon_{i j} t^{i} t^{j}+\cdots+\epsilon_{i_{1} \cdots i_{N}}\left(t^{1}\right)^{i_{1}} \cdots\left(t^{N}\right)^{i_{N}}+\cdots,
$$

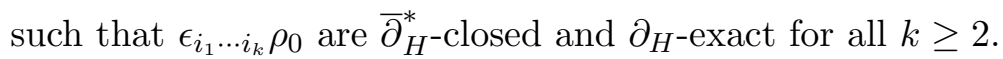


For simplicity the notation, let us assume that $\operatorname{dim}_{\mathbb{C}} H_{d_{E}}^{2}\left(M, \wedge^{*} E^{*}\right)=1$ and a basis of $H_{d_{E}}^{2}\left(M, \wedge^{*} E^{*}\right):\left\{\epsilon_{1}\right\}$. So we shall discuss $\epsilon(t)$ in the form of

$$
\epsilon(t):=\epsilon_{1} t+\epsilon_{2} t^{2}+\cdots,
$$

such that $\epsilon_{k} \rho_{0}$ be $\bar{\partial}_{H}^{*}$-closed and $\partial_{H}$-exact for all $k \geq 2$.

To compare the coefficients of $t$ in the Maurer-Cartan equation, we get

$$
\begin{aligned}
k=1, \bar{\partial}_{H}\left(\epsilon_{1} \rho_{0}\right) & =0, \\
k=2, \bar{\partial}_{H}\left(\epsilon_{2} \rho_{0}\right) & =-\frac{1}{2}\left[\epsilon_{1}, \epsilon_{1}\right]_{H} \rho_{0}, \\
& \cdots \\
k, \bar{\partial}_{H}\left(\epsilon_{k} \rho_{0}\right) & =-\frac{1}{2} \sum_{i=1}^{k-1}\left[\epsilon_{i}, \epsilon_{k-i}\right]_{H} \rho_{0} .
\end{aligned}
$$

Let

$$
\psi_{k}:=-\frac{1}{2} \sum_{i=1}^{k-1}\left[\epsilon_{i}, \epsilon_{k-i}\right]_{H} \rho_{0} .
$$

We show that $\bar{\partial}_{H} \psi_{k}=0$ by induction on $k$.

For $k=2$, we have

$$
\begin{aligned}
\bar{\partial}_{H} \psi_{2} & :=\bar{\partial}_{H}\left(-\frac{1}{2}\left[\epsilon_{1}, \epsilon_{1}\right]_{H} \rho_{0}\right) \\
& =-\frac{1}{2}\left(d_{E}\left[\epsilon_{1}, \epsilon_{1}\right]_{H}\right) \rho_{0} \\
& =-\frac{1}{2}\left(\left[d_{E} \epsilon_{1}, \epsilon_{1}\right]_{H}-\left[\epsilon_{1}, d_{E} \epsilon_{1}\right]_{H}\right) \rho_{0} \\
& =0
\end{aligned}
$$

The last equality holds since $\epsilon_{1} \in H_{d_{E}}^{2}\left(M, \wedge^{*} E^{*}\right)$, hence $d_{E} \epsilon_{1}=0$. 
Some Results of Deformations on Compact $H$-twisted Generalized...151

If we suppose that $i$ from 2 to $k-1, \bar{\partial}_{H} \psi_{i}=0$ holds. For $i=k$, we have

$$
\begin{aligned}
& -2 \bar{\partial}_{H} \psi_{k} \\
:= & \bar{\partial}_{H}\left(\sum_{i=1}^{k-1}\left[\epsilon_{i}, \epsilon_{k-i}\right]_{H} \rho_{0}\right) \\
= & \sum_{i=1}^{k-1}\left(d_{E}\left[\epsilon_{i}, \epsilon_{k-i}\right]_{H}\right) \rho_{0} \\
= & \sum_{i=1}^{k-1}\left(\left[d_{E} \epsilon_{i}, \epsilon_{k-i}\right]_{H}-\left[\epsilon_{i}, d_{E} \epsilon_{k-i}\right]_{H}\right) \rho_{0} \\
= & -\frac{1}{2} \sum_{i=1}^{k-1} \sum_{j=1}^{i-1}\left(\left[\left[\epsilon_{j}, \epsilon_{i-j}\right]_{H}, \epsilon_{k-i}\right]_{H}\right) \rho_{0}+\frac{1}{2} \sum_{i=1}^{k-1} \sum_{j=1}^{i-j-1}\left(\left[\epsilon_{i},\left[\epsilon_{j}, \epsilon_{n-i-j}\right]_{H}\right) \rho_{0}\right. \\
= & \frac{1}{2} \sum_{i=1}^{k-1} \sum_{j=1}^{i-1}\left(\left[\epsilon_{k-i},\left[\epsilon_{j}, \epsilon_{i-j}\right]_{H}\right]_{H}\right) \rho_{0}+\frac{1}{2} \sum_{i=1}^{k-1} \sum_{j=1}^{i-j-1}\left(\left[\epsilon_{i},\left[\epsilon_{j}, \epsilon_{n-i-j}\right]_{H}\right) \rho_{0}\right. \\
= & 2 \sum_{i+j+h=k, 1 \leq i<j<h}\left(\left[\epsilon_{i},\left[\epsilon_{j}, \epsilon_{h}\right]_{H}\right]_{H}+\left[\epsilon_{j},\left[\epsilon_{h}, \epsilon_{i}\right]_{H}\right]_{H}+\left[\epsilon_{h},\left[\epsilon_{i}, \epsilon_{j}\right]_{H}\right]_{H}\right) \rho_{0} \\
& +\sum_{2 i+j=k, 1 \leq i \neq j}\left(2\left[\epsilon_{i},\left[\epsilon_{i}, \epsilon_{j}\right]_{H}\right]_{H}+\left[\epsilon_{j},\left[\epsilon_{i}, \epsilon_{i}\right]_{H}\right]_{H}\right) \rho_{0} \\
& +\sum_{3 i=k, 1 \leq i}\left[\epsilon_{i},\left[\epsilon_{i}, \epsilon_{i}\right]_{H}\right]_{H} \rho_{0} \\
= & 0
\end{aligned}
$$

The 2nd equality holds by the definition of $[\cdot, \cdot]_{H}$ and $d_{E}$; the 3 rd equality is the induction; and the last equality holds since the Jacobian identity. See P21 in [2].

Next, we show that $\psi_{k}$ is $\partial_{H}$-exact by induction on $k$, and there exists an unique $\epsilon_{k} \rho_{0}$, such that $\epsilon_{k} \rho_{0}$ is $\partial_{H^{-}}$-exact, $\bar{\partial}_{H^{-}}^{*}$ closed for any $k \geq 2$.

Since $\epsilon_{1} \in H_{d_{E}}^{2}\left(M, \wedge^{*} E^{*}\right)$, in Calabi-Yau case, we have $\epsilon_{1} \rho_{0} \in$ $H_{\bar{\partial}_{H}}^{2}\left(M, \wedge^{*} T_{M}^{*}\right)$. Hence $\triangle_{\bar{\partial}_{H}}\left(\epsilon_{1} \rho_{0}\right)=\triangle_{\partial_{H}}\left(\epsilon_{1} \rho_{0}\right)=0$ since $\triangle_{\partial_{H}}=\triangle_{\bar{\partial}_{H}}$. So we get $\partial_{H}\left(\epsilon_{1} \rho_{0}\right)=0$ (We need this since it is the condition of the formula (3.1) in Lemma 3.2.).

Therefore, for $k=2$, by using the formula (3.1) in Lemma 3.2 , we have

$$
\begin{aligned}
\psi_{2} & :=-\frac{1}{2}\left[\epsilon_{1}, \epsilon_{1}\right]_{H} \\
& =\frac{1}{2} \partial_{H}\left(\epsilon_{1} \epsilon_{1} \rho_{0}\right),
\end{aligned}
$$


i.e. $\psi_{2}$ is $\partial_{H}$-exact.

Since

$$
\begin{aligned}
\frac{1}{2} \bar{\partial}_{H} \partial_{H}\left(\epsilon_{1} \epsilon_{1} \rho_{0}\right) & :=\bar{\partial}_{H} \psi_{2} \\
& =0
\end{aligned}
$$

by Proposition 4.2 , we can get that the equation

$$
\bar{\partial}_{H} \epsilon_{2} \rho_{0}=\frac{1}{2} \partial_{H} \epsilon_{1} \epsilon_{1} \rho_{0}
$$

has the unique solution

$$
\epsilon_{2} \rho_{0}=\frac{1}{2} \bar{\partial}_{H}^{*} G \partial_{H}\left(\epsilon_{1} \epsilon_{1} \rho_{0}\right) .
$$

which satisfying that $\epsilon_{2} \rho_{0}$ is $\bar{\partial}_{H}^{*}$-closed and $\partial_{H}$-exact thus $\partial_{H}$-closed.

If we suppose that for $i=2$ to $k-1, \epsilon_{i} \rho_{0}$ exists uniquely, such that $\epsilon_{i} \rho_{0}$ is $\partial_{H}$-exact, $\bar{\partial}_{H}^{*}$-closed hold. For $i=k$, we have

$$
\psi_{k}:=-\frac{1}{2} \sum_{i=1}^{k-1}\left[\epsilon_{i}, \epsilon_{k-i}\right]_{H} \rho_{0}=\frac{1}{2} \partial_{H}\left(\sum_{i=1}^{k-1} \epsilon_{i} \epsilon_{k-i} \rho_{0}\right)
$$

is also $\partial_{H \text {-exact. }}$

Since

$$
\begin{aligned}
\frac{1}{2} \bar{\partial}_{H} \partial_{H}\left(\sum_{i=1}^{k-1} \epsilon_{i} \epsilon_{k-i} \rho_{0}\right) & :=\bar{\partial}_{H} \psi_{k} \\
& =0,
\end{aligned}
$$

by Proposition 4.2 , we can get that the equation

$$
\bar{\partial}_{H} \epsilon_{k} \rho_{0}=\frac{1}{2} \partial_{H} \sum_{i=1}^{k-1} \epsilon_{i} \epsilon_{k-i} \rho_{0}
$$

has the solution

$$
\epsilon_{k} \rho_{0}=\frac{1}{2} \bar{\partial}_{H}^{*} G \partial_{H}\left(\sum_{i=1}^{k-1} \epsilon_{i} \epsilon_{k-i} \rho_{0}\right) .
$$

(Since $U_{i}:=\wedge^{i} E^{*} \cdot U_{0}$ and $U_{0}$ is a complex line bundle whose generator is $\rho_{0}$, any $\alpha \in U_{i}$ can be represented as $A \rho_{0}$, where $A \in \wedge^{i} E^{*}$.) 
Some Results of Deformations on Compact $H$-twisted Generalized...153

Hence by Proposition 4.2, we can get that $\mathbb{H}\left(\epsilon_{k} \rho_{0}\right)=0$ and $\epsilon_{k} \rho$ is $\partial_{H^{-}}$ exact, $\bar{\partial}_{H^{-}}^{*}$ closed, and

$$
\left\|\epsilon_{k} \rho_{0}\right\|^{2} \leq \frac{1}{4}\left(\partial_{H}\left(\sum_{i=1}^{k-1} \epsilon_{i} \epsilon_{k-i} \rho\right), G \partial_{H}\left(\sum_{i=1}^{k-1} \epsilon_{i} \epsilon_{k-i} \rho_{0}\right)\right) .
$$

For the general case of $t=\left(t^{1}, \cdots, t^{N}\right)$, we can also give the same discussion.

Comparing the coefficients of $t=\left(t^{1}, \cdots, t^{N}\right)$ in the Maurer-Cartan equation, we get

$$
\begin{aligned}
k=1, \bar{\partial}_{H}\left(\epsilon_{i} \rho_{0}\right)= & 0, \\
k=2, \bar{\partial}_{H}\left(\epsilon_{i j} \rho_{0}\right)= & -\frac{1}{2}\left(\left[\epsilon_{i}, \epsilon_{j}\right]_{H}+\left[\epsilon_{j}, \epsilon_{i}\right]_{H}\right) \rho_{0}, \\
\cdots & \sum_{k, \bar{\partial}_{H}\left(\epsilon_{i_{1} \cdots i_{N}} \rho_{0}\right)=}-\frac{1}{2} \sum_{j_{\alpha}+h_{\alpha}=i_{\alpha}, j_{\alpha}, h_{\alpha} \geq 0, \alpha=1 \cdots N}\left[\epsilon_{j_{1} \cdots j_{N}}, \epsilon_{h_{1} \cdots h_{N}}\right]_{H} \rho_{0} . \\
& \left(i_{1}+\cdots+i_{N}=k\right)
\end{aligned}
$$

Let

$$
\psi_{i_{1} \cdots i_{N}}:=-\frac{1}{2} \sum_{j_{\alpha}+h_{\alpha}=i_{\alpha}, j_{\alpha}, h_{\alpha} \geq 0, \alpha=1 \cdots N}\left[\epsilon_{j_{1} \ldots j_{N}}, \epsilon_{h_{1} \cdots h_{N}}\right]_{H} \rho_{0} .
$$

We show that $\bar{\partial}_{H} \psi_{i_{1} \ldots i_{N}}=0$ by induction on $i_{1}+\ldots+i_{N}=k$.

For $k=2$, we have

$$
\begin{aligned}
\bar{\partial}_{H} \psi_{i j}:= & \bar{\partial}_{H}\left(-\frac{1}{2}\left(\left[\epsilon_{i}, \epsilon_{j}\right]_{H}+\left[\epsilon_{j}, \epsilon_{i}\right]_{H}\right) \rho_{0}\right) \\
= & -\frac{1}{2}\left(d_{E}\left(\left[\epsilon_{i}, \epsilon_{j}\right]_{H}+\left[\epsilon_{j}, \epsilon_{i}\right]_{H}\right)\right) \rho_{0} \\
= & -\frac{1}{2}\left(\left[d_{E} \epsilon_{i}, \epsilon_{j}\right]_{H}-\left[\epsilon_{i}, d_{E} \epsilon_{j}\right]_{H}\right)+\left[d_{E} \epsilon_{j}, \epsilon_{i}\right]_{H} \\
& \left.-\left[\epsilon_{j}, d_{E} \epsilon_{i}\right]_{H}\right) \rho_{0} \\
= & 0 .
\end{aligned}
$$

The last equality holds since $\epsilon_{i} \in H^{2}\left(M, \wedge^{*} E^{*}\right)$, hence $d_{E} \epsilon_{i}=0$. 
If we suppose that from $i_{1}+\cdots+i_{N}=2$ to $k-1, \bar{\partial}_{H} \psi_{i_{1} \cdots i_{N}}=0$ holds. For $i_{1}+\cdots+i_{N}=k$, we have

$$
\begin{aligned}
& -2 \bar{\partial}_{H} \psi_{i_{1} \cdots i_{N}} \\
& :=\bar{\partial}_{H}\left(\sum_{j_{\alpha}+h_{\alpha}=i_{\alpha}, j_{\alpha}, h_{\alpha} \geq 0, \alpha=1 \cdots N}\left[\epsilon_{j_{1} \cdots j_{N}}, \epsilon_{h_{1} \cdots h_{N}}\right]_{H} \rho_{0}\right) \\
& =\sum_{j_{\alpha}+h_{\alpha}=i_{\alpha}, j_{\alpha}, h_{\alpha} \geq 0, \alpha=1 \cdots N}\left(d_{E}\left(\left[\epsilon_{j_{1} \cdots j_{N}}, \epsilon_{h_{1} \cdots h_{N}}\right]_{H}\right)\right) \rho_{0} \\
& \left.\left.=\sum_{j_{\alpha}+h_{\alpha}=i_{\alpha}, j_{\alpha}, h_{\alpha} \geq 0, \alpha=1 \cdots N}\left(\left[d_{E} \epsilon_{j_{1} \cdots j_{N}}, \epsilon_{h_{1} \cdots h_{N}}\right]_{H}\right)-\left[\epsilon_{j_{1} \cdots j_{N}}, d_{E} \epsilon_{h_{1} \cdots h_{N}}\right]_{H}\right)\right) \rho_{0} \\
& =2 \sum_{j_{\alpha}+h_{\alpha}+p_{\alpha}=i_{\alpha}}\left(\left[\epsilon_{j_{1} \cdots j_{N}},\left[\epsilon_{h_{1} \cdots h_{N}}, \epsilon_{p_{1} \cdots p_{N}}\right]_{H}\right]_{H}+\right. \\
& \left.\left[\epsilon_{h_{1} \cdots h_{N}},\left[\epsilon_{p_{1} \cdots p_{N}}, \epsilon_{j_{1} \cdots j_{N}}\right]_{H}\right]_{H}+\left[\epsilon_{p_{1} \cdots p_{N}},\left[\epsilon_{j_{1} \cdots j_{N}}, \epsilon_{h_{1} \cdots h_{N}}\right]_{H}\right]_{H}\right) \rho_{0} \\
& +\sum_{2 j_{\alpha}+h_{\alpha}=i_{\alpha}}\left(2\left[\epsilon_{j_{1} \cdots j_{N}},\left[\epsilon_{j_{1} \cdots j_{N}}, \epsilon_{h_{1} \cdots h_{N}}\right]_{H}\right]_{H}\right. \\
& \left.+\left[\epsilon_{h_{1} \cdots h_{N}},\left[\epsilon_{j_{1} \cdots j_{N}}, \epsilon_{j_{1} \cdots j_{N}}\right]_{H}\right]_{H}\right) \rho_{0}+\sum_{3 j_{\alpha}=i_{\alpha}}\left[\epsilon_{j_{1} \cdots j_{N}},\left[\epsilon_{j_{1} \cdots j_{N}}, \epsilon_{j_{1} \cdots j_{N}}\right]_{H}\right]_{H} \rho_{0} \\
& =0
\end{aligned}
$$

The 2nd equality holds by the definition of $[\cdot, \cdot]_{H}$ and $d_{E}$; the 3rd equality is the induction; and the last equality holds since the Jacobian identity.

Next, we show that $\psi_{i_{1} \cdots i_{N}}$ is $\partial_{H^{-}}$-exact by induction on $i_{1}+\cdots+i_{N}=k$, and there exists an unique $\epsilon_{i_{1} \ldots i_{N}} \rho_{0}$, such that $\epsilon_{i_{1} \ldots i_{N}} \rho_{0}$ is $\partial_{H^{-}}$-exact, $\bar{\partial}_{H^{-}}^{*}$ closed for any $i_{1}+\cdots+i_{N}=k \geq 2$.

Since $\epsilon_{i} \in H_{d_{E}}^{2}\left(M, \wedge^{*} E^{*}\right)$, in Calabi-Yau case, we have $\epsilon_{i} \rho_{0} \in$ $H_{\bar{\partial}_{H}}^{2}\left(M, \wedge^{*} T_{M}^{*}\right)$. Hence $\triangle_{\bar{\partial}_{H}}\left(\epsilon_{i} \rho_{0}\right)=\triangle_{\partial_{H}}\left(\epsilon_{i} \rho_{0}\right)=0$ since $\triangle_{\partial_{H}}=\triangle_{\bar{\partial}_{H}}$. So we get $\partial_{H}\left(\epsilon_{i} \rho_{0}\right)=0$.

Therefore, for $k=2$, by using the formula (3.1) in Lemma 3.2, we have

$$
\begin{aligned}
\psi_{i j} & :=-\frac{1}{2}\left(\left[\epsilon_{i}, \epsilon_{j}\right]_{H}+\left[\epsilon_{i}, \epsilon_{j}\right]_{H}\right) \\
& =\frac{1}{2} \partial_{H}\left(\left(\epsilon_{i} \epsilon_{j}+\epsilon_{j} \epsilon_{i}\right) \rho_{0}\right)
\end{aligned}
$$

i.e. $\psi_{i j}$ is $\partial_{H}$-exact.

Since

$$
\begin{aligned}
\frac{1}{2} \bar{\partial}_{H} \partial_{H}\left(\epsilon_{i} \epsilon_{j} \rho_{0}+\epsilon_{j} \epsilon_{i} \rho_{0}\right) & :=\bar{\partial}_{H} \psi_{i j} \\
& =0
\end{aligned}
$$


Some Results of Deformations on Compact $H$-twisted Generalized...155

by Proposition 4.2 , we can get that the equation

$$
\bar{\partial}_{H} \epsilon_{i j} \rho_{0}=\frac{1}{2} \partial_{H}\left(\epsilon_{i} \epsilon_{j}+\epsilon_{j} \epsilon_{i}\right) \rho_{0}
$$

has the unique solution

$$
\epsilon_{i j} \rho_{0}=\frac{1}{2} \bar{\partial}_{H}^{*} G \partial_{H}\left(\left(\epsilon_{i} \epsilon_{j}+\epsilon_{j} \epsilon_{i}\right) \rho_{0}\right) .
$$

which satisfying that $\epsilon_{i j} \rho_{0}$ is $\bar{\partial}_{H^{-}}^{*}$ closed and $\partial_{H^{-}}$exact.

If we suppose that for $i_{1}+\cdots+i_{N}=2$ to $k-1, \epsilon_{i_{1} \cdots i_{N}} \rho_{0}$ exists uniquely, such that $\epsilon_{i_{1} \cdots i_{N}} \rho_{0}$ is $\partial_{H}$-exact, $\bar{\partial}_{H^{-}}^{*}$-closed hold. For $i_{1}+\cdots+i_{N}=k$, we have

$$
\begin{aligned}
\psi_{i_{1} \cdots i_{N}} & :=-\frac{1}{2} \sum_{j_{\alpha}+h_{\alpha}=i_{\alpha}}\left[\epsilon_{j_{1} \cdots j_{N}}, \epsilon_{h_{1} \cdots h_{N}}\right]_{H} \rho_{0} \\
& =\frac{1}{2} \partial_{H}\left(\sum_{j_{\alpha}+h_{\alpha}=i_{\alpha}} \epsilon_{j_{1} \cdots j_{N}} \epsilon_{h_{1} \cdots h_{N}} \rho_{0}\right)
\end{aligned}
$$

is also $\partial_{H}$-exact.

Since

$$
\begin{aligned}
\frac{1}{2} \bar{\partial}_{H} \partial_{H}\left(\sum_{j_{\alpha}+h_{\alpha}=i_{\alpha}} \epsilon_{j_{1} \cdots j_{N}} \epsilon_{h_{1} \cdots h_{N}} \rho_{0}\right) & :=\bar{\partial}_{H} \psi_{i_{1} \cdots i_{N}} \\
& =0
\end{aligned}
$$

by Proposition 4.2 , we can get that the equation

$$
\bar{\partial}_{H} \epsilon_{i_{1} \cdots i_{N}} \rho_{0}=\frac{1}{2} \partial_{H} \sum_{j_{\alpha}+h_{\alpha}=i_{\alpha}} \epsilon_{j_{1} \cdots j_{N}} \epsilon_{h_{1} \cdots h_{N}} \rho_{0}
$$

has the unique solution

$$
\epsilon_{i_{1} \cdots i_{N}} \rho_{0}=\frac{1}{2} \bar{\partial}_{H}^{*} G \partial_{H}\left(\sum_{j_{\alpha}+h_{\alpha}=i_{\alpha}} \epsilon_{j_{1} \cdots j_{N}} \epsilon_{h_{1} \cdots h_{N}} \rho_{0}\right) .
$$

And by Proposition 4.2 we can get that $\mathbb{H}\left(\epsilon_{i_{1} \cdots i_{N}} \rho_{0}\right)=$ 0, $\epsilon_{i_{1} \cdots i_{N}} \rho \quad$ is $\partial_{H}$-exact, $\quad \bar{\partial}_{H}^{*}$-closed, and $\left\|\epsilon_{i_{1} \cdots i_{N}} \rho_{0}\right\|^{2} \leq$ $\frac{1}{4}\left(\partial_{H}\left(\sum_{j_{\alpha}+h_{\alpha}=i_{\alpha}} \epsilon_{j_{1} \cdots j_{N}} \epsilon_{h_{1} \cdots h_{N}} \rho_{0}\right), G \partial_{H}\left(\sum_{j_{\alpha}+h_{\alpha}=i_{\alpha}} \epsilon_{j_{1} \cdots j_{N}} \epsilon_{h_{1} \cdots h_{N}} \rho_{0}\right)\right)$.

Step 2: We show $\epsilon(t) L^{2}$ converges at $|t| \leq \frac{1}{4 a c}$ by using the same convergent power series as in the [12]. 
Firstly, we have

$$
\begin{aligned}
\left\|\epsilon_{k_{1} \cdots k_{N}} \rho_{0}\right\| & :=\frac{1}{2}\left\|\bar{\partial}_{H} G \partial_{H}^{*}\left(\sum_{i_{\alpha}+j_{\alpha}=k_{\alpha}} \epsilon_{i_{1} \cdots i_{N}} \epsilon_{j_{1} \ldots j_{N}} \rho_{0}\right)\right\| \\
& \leq \sum_{i_{\alpha}+j_{\alpha}=k_{\alpha}}\left\|\epsilon_{i_{1} \cdots i_{N}} \epsilon_{j_{1} \cdots j_{N}} \rho_{0}\right\| \\
& \leq \sum_{i_{\alpha}+j_{\alpha}=k_{\alpha}}\left\|\epsilon_{i_{1} \cdots i_{N}} \rho_{0}\right\|_{s+\alpha} \cdot\left\|\epsilon_{j_{1} \cdots j_{N}} \rho_{0}\right\|,
\end{aligned}
$$

where $k \geq 2 \in \mathbb{N}, 0 \leq \alpha \leq 1$. The 1 st inequality holds since Lemma 4.1 and the last inequality holds by the definition of the Hölder norms.

Secondly, we set $\left\{x_{i}\right\}_{i=1}^{\infty}$, such that $x_{1}=a, x_{k}=c \sum_{i=1}^{k-1} x_{i} x_{k-i}$ just as in Lemma 3.4 and $c$ is a constant definite in inequality (3.2) after Lemma 3.3. We set $c:=\max \{1, c\}$ which we still denote it as $c$. We will show that

$$
x_{k} \geq \sum_{k_{1}+\cdots+k_{N}=k}\left\|\epsilon_{k_{1} \cdots k_{N}} \rho_{0}\right\|_{s+\alpha}
$$

by induction on $k$.

Since $\rho_{0}, \epsilon_{i}, s \geq 2, \alpha$ fixed, we can assume that $a=$ $\max \left\{\sum_{i=1}^{N}\left\|\epsilon_{i} \rho_{0}\right\|, \sum_{i=1}^{N}\left\|\epsilon_{i} \rho_{0}\right\|_{s+\alpha},\left\|\rho_{0}\right\|\right\}$ is a constant.

For $k=2$, we have

$$
\begin{aligned}
x_{2} & :=c x_{1} x_{1} \\
& =c\left(\sum_{i=1}^{N}\left\|\epsilon_{i} \rho_{0}\right\|_{s+\alpha}\right)\left(\sum_{i=1}^{N}\left\|\epsilon_{i} \rho_{0}\right\|_{s+\alpha}\right) \\
& \geq \frac{1}{2} \sum_{i, j=1}^{N}\left\|\bar{\partial}_{H}^{*} G \partial_{H}\left(\epsilon_{i} \epsilon_{j} \rho_{0}\right)\right\|_{s+\alpha} \\
& \geq \sum_{i, j=1}^{N}\left\|\epsilon_{i j} \rho_{0}\right\|_{s+\alpha} .
\end{aligned}
$$

The 1st inequality holds since Lemma 3.3 , and the last inequality holds since the construction of $\epsilon(t)$

If for $\leq k-1$, the inequality above holds, for $k$, we have 
Some Results of Deformations on Compact $H$-twisted Generalized...157

$$
\begin{aligned}
x_{k} & :=c \sum_{i+j=k} x_{i} x_{j} \\
& \geq c \sum_{i+j=k}\left(\sum_{i_{1}+\cdots+i_{N}=i}\left\|\epsilon_{i_{1} \cdots i_{N}} \rho_{0}\right\|_{s+\alpha}\right)\left(\sum_{j_{1}+\cdots+j_{N}=j}\left\|\epsilon_{j_{1} \cdots j_{N}} \rho_{0}\right\|_{s+\alpha}\right) \\
& \geq c \sum_{i+j=k}\left(\sum_{i_{1}+\cdots+i_{N}=i}\left\|\epsilon_{i_{1} \cdots i_{N}}\right\|_{s+\alpha}\right)\left(\sum_{j_{1}+\cdots+j_{N}=j}\left\|\epsilon_{j_{1} \cdots j_{N}} \rho_{0}\right\|_{s+\alpha}\right) \\
& =\frac{1}{2} \sum_{i_{1}+\cdots+i_{N}+j_{1}+\cdots+j_{N}=k}\left\|\bar{\partial}_{H}^{*} G \partial_{H}\left(\epsilon_{i_{1} \cdots i_{N}} \epsilon_{j_{1} \cdots j_{N}} \rho_{0}\right)\right\|_{s+\alpha} \\
(5.2) & \geq \sum_{k_{1}+\cdots+k_{N}=k}\left\|\epsilon_{k_{1} \cdots k_{N}} \rho_{0}\right\|_{s+\alpha} .
\end{aligned}
$$

The 1st inequality holds since the induction, the 2nd equality holds since $\rho_{0}$ is fixed and we consider $\left\|\epsilon_{i_{1} \cdots i_{N}} \rho_{0}\right\|_{s+\alpha}$ equivalent to $\left\|\epsilon_{i_{1} \cdots i_{N}}\right\|_{s+\alpha}$, and the constant $c^{\prime}$ is different, for simplity, we consider $\max \left\{c, c^{\prime}\right\}$ and still denote as $c$.

We also need to show

$$
x_{k} \geq \sum_{k_{1}+\cdots+k_{N}=k}\left\|\epsilon_{k_{1} \cdots k_{N}} \rho_{0}\right\|
$$

by induction on $k$.

For $k=2$, we have

$$
\begin{aligned}
x_{2} & :=c x_{1} x_{1} \\
& =c\left(\sum_{i=1}^{N}\left\|\epsilon_{i} \rho_{0}\right\|_{s+\alpha}\right)\left(\sum_{i=1}^{N}\left\|\epsilon_{i} \rho_{0}\right\|\right) \\
& \geq \sum_{i, j=1}^{N}\left\|\epsilon_{i j} \rho_{0}\right\| .
\end{aligned}
$$

If for $\leq k-1$, the inequality above holds, for $k$, we have 


$$
\begin{aligned}
x_{k} & :=c \sum_{i+j=k} x_{i} x_{j} \\
& \geq c \sum_{i+j=k}\left(\sum_{i_{1}+\cdots+i_{N}=i}\left\|\epsilon_{i_{1} \cdots i_{N}} \rho_{0}\right\|_{s+\alpha}\right)\left(\sum_{j_{1}+\cdots+j_{N}=j}\left\|\epsilon_{j_{1} \cdots j_{N}} \rho_{0}\right\|\right) \\
& \geq \frac{1}{2} \sum_{i_{1}+\cdots+i_{N}+j_{1}+\cdots+j_{N}=k}\left\|\bar{\partial}_{H}^{*} G \partial_{H} \epsilon_{i_{1} \cdots i_{N}} \epsilon_{j_{1} \cdots j_{N}} \rho_{0}\right\| \\
& \geq \sum_{k_{1}+\cdots+k_{N}=k}\left\|\epsilon_{k_{1} \cdots k_{N}} \rho_{0}\right\|
\end{aligned}
$$

by formula (5.1).

Hence, we get

$$
\begin{aligned}
& \left\|\epsilon(t) \rho_{0}\right\| \\
:= & \left\|\sum_{i=1}^{N} \epsilon_{i} \rho_{0} t^{i}+\sum_{k \geq 2, k_{1}+\cdots+k_{N}=k} \epsilon_{k_{1} \cdots k_{N}} \rho_{0}\left(t^{1}\right)^{k_{1}} \cdots\left(t^{N}\right)^{k_{N}}\right\| \\
:= & \left\|\sum_{i=1}^{N} \epsilon_{i} \rho_{0}\right\| \cdot\left|t^{i}\right|+\frac{1}{2} \sum_{k \geq 2, k_{1}+\cdots+k_{N}=k} \| \partial_{H}^{*} G \partial_{H} \sum_{i_{\alpha}+j_{\alpha}=k_{\alpha}} \epsilon_{i_{1} \cdots i_{N}} \\
& \epsilon_{j_{1} \cdots j_{N}} \rho_{0}\left(t^{1}\right)^{k_{1}} \cdots\left(t^{N}\right)^{k_{N}} \| \\
\leq & \sum_{i=1}^{N}\left\|\epsilon_{i} \rho_{0}\right\| \cdot\left|t^{i}\right|+\frac{1}{2} \sum_{k \geq 2, k_{1}+\cdots+k_{N}=k}\left\|\sum_{i_{\alpha}+j_{\alpha}=k_{\alpha}} \epsilon_{i_{1} \cdots i_{N}} \epsilon_{j_{1} \cdots j_{N}} \rho_{0}\right\| \\
\leq & \quad\left|t^{1}\right|^{k_{1}} \cdots|t|+c\left|t^{N}\right|^{k_{N}} \sum_{k \geq 2, i_{1}+\cdots+i_{N}+j_{1}+\cdots+j_{N}=k}\left\|\epsilon_{i_{1} \cdots i_{N}} \rho_{0}\right\|\left\|_{s+\alpha} \cdot\right\| \epsilon_{j_{1} \cdots j_{N}} \rho_{0} \| \cdot|t|^{k} \\
& \left(t=\max _{i}\left\{t^{i}\right\}\right) \\
\leq & x_{1}|t|+\sum_{k \geq 2} x_{k}|t|^{k} \\
< & +\infty
\end{aligned}
$$

at $|t| \leq \frac{1}{4 a c}$. 
Some Results of Deformations on Compact $H$-twisted Generalized...159

Remark 5.2. (1)Since

$$
\begin{aligned}
\bar{\partial}_{H}\left(\epsilon_{i_{1} \cdots i_{N}} \rho_{0}\right)= & -\frac{1}{2} \sum_{j_{\alpha}+h_{\alpha}=i_{\alpha}, j_{\alpha}, h_{\alpha} \geq 0, \alpha=1 \cdots N}\left[\epsilon_{j_{1} \cdots j_{N}}, \epsilon_{h_{1} \cdots h_{N}}\right]_{H} \rho_{0}, \\
& \left(i_{1}+\cdots+i_{N}=k\right) \\
\bar{\partial}_{H}^{*}\left(\epsilon_{i_{1} \cdots i_{N}} \rho_{0}\right)= & 0
\end{aligned}
$$

we can get

$$
\begin{aligned}
\triangle_{\bar{\partial}_{H}}\left(\epsilon_{i_{1} \cdots i_{N}} \rho_{0}\right) & =\bar{\partial}_{H}^{*} \bar{\partial}_{H}\left(\epsilon_{i_{1} \cdots i_{N}} \rho_{0}\right) \\
& =-\frac{1}{2} \sum_{j_{\alpha}+h_{\alpha}=i_{\alpha}, j_{\alpha}, h_{\alpha} \geq 0, \alpha=1 \cdots N} \bar{\partial}_{H}^{*}\left(\left[\epsilon_{j_{1} \cdots j_{N}}, \epsilon_{h_{1} \cdots h_{N}}\right]_{H} \rho_{0}\right) .
\end{aligned}
$$

So by the assumption that $\epsilon_{i} \rho_{0}$ is smooth on $M$, and $\triangle_{\bar{\partial}_{H}}$ is a strongly elliptic operator, we can get that $\epsilon_{i_{1} \cdots i_{N}} \rho_{0}$ is smooth on $M$ by the elliptic regularity theorem in [17], and the induction on $i_{1}+\cdots+i_{N}=k$.

(2)From P281 in [11], we have known that $\epsilon(t) \rho_{0}$ is smooth on $M \times \triangle_{\delta}$ for a sufficiently small neighbourhood of $t \in \triangle_{\delta}$.

\section{Global canonical family}

In this section, if we assume that the deformation is smooth in a fixed neighbourhood, we shall get the global canonical sections for deformations of compact $H$-twisted Generalized Kähler manifold by using the parallel method in 12 and [22] if we assume the existence of a global canonical family of deformation. There are already many works on [18, 19.

First, we have the following equivalence to criterion the holomorphism with respect to the generalized complex structure.

Proposition 6.1. [20] On compact generalized Kähler manifold M. For any $\rho \in U_{0, M}, e^{-\epsilon(t)} \rho$ is holomorphic with respect to the generalized complex structure $J_{\epsilon(t)}$ induced by $\epsilon(t)$ on $M_{t}$ if and only if

$$
\bar{\partial}_{H} \rho-\partial_{H}(\epsilon(t) \rho)=0 .
$$

Proof. Since if $A \in E$,

$$
((1+\epsilon) A) \rho=e^{-\epsilon} A e^{\epsilon} \rho,
$$


(See P16, Proposition 7 in [14].) we have

$$
\begin{aligned}
((1+\epsilon) A) e^{-\epsilon} \rho_{0} & =e^{-\epsilon} A e^{\epsilon} e^{-\epsilon} \rho \\
& =e^{-\epsilon} A \rho \\
& =0 .
\end{aligned}
$$

The last equality is by the definition of $U_{0}$. Hence we get $e^{-\epsilon(t)} \rho \in U_{0, M_{t}}$.

Since

$$
e^{\epsilon} d_{H} e^{-\epsilon} \rho=\bar{\partial}_{H} \rho-\partial_{H}(\epsilon \rho)
$$

we have

$$
d_{H} e^{-\epsilon} \rho=0 \Leftrightarrow \bar{\partial}_{H} \rho-\partial_{H}(\epsilon \rho)=0 .
$$

Since $e^{-\epsilon} \rho \in U_{0, M_{t}}$, we have

$$
\partial_{H, t} e^{-\epsilon} \rho=0
$$

where $d_{H}=\partial_{H, t}+\bar{\partial}_{H, t}$ on $M_{t}$. So we have

$$
\bar{\partial}_{H, t} e^{-\epsilon} \rho=0 \Leftrightarrow \bar{\partial}_{H} \rho-\partial_{H}(\epsilon \rho)=0 .
$$

In compact generalized Calabi-Yau case, we have

Proposition 6.2 (See P16, Proposition 7 in [14].). If we assume that $\epsilon(t)$ smooth with fixed convergence radius exists, then

$$
\epsilon(t) \rho_{0} \in H_{\bar{\partial}_{H}}^{2}\left(M, \wedge^{*} T_{M}^{*}\right),
$$

where $H_{\bar{\partial}_{H}}^{2}\left(M, \wedge^{*} T_{M}^{*}\right)$ is the cohomology group of the complex $\left(\wedge^{*} T_{M}^{*}, \bar{\partial}_{H}\right)$, and

$$
\rho_{t}^{c}:=e^{-\epsilon(t)} \rho_{0}
$$

holomorphic with respect to $J_{\epsilon(t)}$ with fixed convergence radius. 
Some Results of Deformations on Compact $H$-twisted Generalized...161

Proof. For any test form $\eta$ on $M$,

$$
\begin{aligned}
\left(\partial_{H}\left(\epsilon(t) \rho_{0}\right), \eta\right) & =\left(\epsilon(t) \rho_{0}, \partial_{H}^{*} \eta\right) \\
& =\lim _{k \rightarrow \infty} \sum_{\|I\| \leq k}\left(\epsilon_{I} t^{I} \rho_{0}, \partial_{H}^{*} \eta\right) \\
& =\sum_{i=1}^{N}\left(\partial_{H} \epsilon_{i} t^{i} \rho_{0}, \eta\right)+\lim _{k \rightarrow \infty} \sum_{2 \leq\|I\| \leq k}\left(\partial_{H} \epsilon_{I} t^{I} \rho_{0}, \eta\right) \\
& =0 .
\end{aligned}
$$

The last equality holds since $\epsilon_{i} \rho_{0}$ are harmonic, and $\epsilon_{I} \rho_{0}$ are $\partial_{H}$-exact for $\|I\|] \geq 2$.

So we have $\partial_{H}\left(\epsilon(t) \rho_{0}\right)=0$ in the distribution sense.

Also, $\bar{\partial}_{H} \rho_{0}=0$, and $\epsilon(t)$ is smooth by assumption, we have $\bar{\partial}_{H} \rho-$ $\partial_{H}(\epsilon(t) \rho)=0$ and by Propositon 6.1, we get the conclusion.

Proposition 6.3. Let $\left(M_{t}, J_{\epsilon(t)}\right)$ be the deformation of a compact $H$-twisted generalized Calabi-Yau manifold $M$, then we have

$$
\left[\rho_{t}^{c}\right]=\left[\rho_{0}\right]+\sum_{i=1}^{N}\left[-\epsilon_{i} \rho_{0}\right] t+O\left(|t|^{2}\right),
$$

where $\left[\rho_{0}\right]$ means a representation in $H_{\bar{\partial}_{H}}^{0}\left(M, \wedge^{*} T_{M}^{*}\right)$, and $O\left(|t|^{2}\right)$ denotes the terms in $H_{\bar{\partial}_{H}}^{4}\left(M, \wedge^{*} T_{M}^{*}\right) \bigoplus \cdots \oplus H_{\bar{\partial}_{H}}^{2 n}\left(M, \wedge^{*} T_{M}^{*}\right)$ of order at least 2 in $t$.

Proof. On convergence radius, we have

$$
\begin{aligned}
{\left[\rho_{t}^{c}\right] } & :=\left[\rho_{0}\right]+\sum_{i=1}^{N}\left[\mathbb{H}\left(-\epsilon_{i} \rho_{0}\right)\right] t+\sum_{I,|I| \geq 2}\left[\mathbb{H}\left((-1)^{|I|} \epsilon_{I} \rho_{0}\right)\right] t^{|I|}+\cdots \\
& =\left[\rho_{0}\right]+\sum_{i=1}^{N}\left[-\epsilon_{i} \rho_{0}\right] t+O\left(|t|^{2}\right)
\end{aligned}
$$

where $t^{I}:=\left(t_{1}\right)^{i_{1}} \ldots\left(t_{N}\right)^{i_{N}}, \epsilon_{I}:=\epsilon_{i_{1} \ldots t_{N}}$.

The last equality holds since $\epsilon_{i} \rho_{0}$ are harmonic, and $\epsilon_{I} \rho_{0}$ are $\partial_{H}$-exact in Calabi-Yau case.

Next, like in [12], as an extension of the case of the generalized CalabiYau manifolds, we assume that on compact generalized Kähler $M$, there 
exists

$$
\epsilon(t):=\epsilon_{i} t^{i}+\epsilon_{i j} t^{i} t^{j}+\cdots+\epsilon_{i_{1} \cdots i_{N}}\left(t^{1}\right)^{i_{1}} \cdots\left(t^{N}\right)^{i_{N}}+\cdots,
$$

satisfying

(1) $\epsilon_{i}$ form a basis of $H_{d_{E}}^{2}\left(M, \wedge^{*} E^{*}\right)$;

(2) the integral condition: $d_{E} \epsilon(t)+\frac{1}{2}[\epsilon(t), \epsilon(t)]_{H}=0$;

(3) $\left\|\epsilon(t) \rho_{0}\right\| \leq \sum_{\|I\|>1}\left\|\epsilon_{I} \mid\right\|\left\|\rho_{0}\right\| t\|I\|<+\infty$ is convergence in $t<\frac{1}{4 a c}$, where for any $\rho_{0} \neq 0 \in H_{\bar{\partial}_{H}}^{0}\left(M, \wedge^{*} T_{M}^{*}\right), x_{k} \geq \sum_{k_{1}+\cdots+k_{N}=k}\left\|\epsilon_{k_{1} \cdots k_{N}}\right\|_{s+\alpha}, x_{k}$ is in Lemma 3.4 and $\epsilon(t)$ has the convergence as the same as that in Theorem 5.1. As an analogue, we can get that

Proposition 6.4. If we assume that $\epsilon(t)$ smooth with convergence radius $\frac{1}{4 a c}$ exists, and $\left(M_{t}, J_{-\epsilon(t)}\right)$ be the deformation, then for any $\rho_{0} \neq 0 \in$ $H_{\bar{\partial}_{H}}^{0}\left(M, \wedge^{*} T_{M}^{*}\right)$, we can construct a smooth power series

$$
\rho_{t}:=\rho_{0}+\sum_{K,|K| \geq 1} \rho_{K} t^{K} \in U_{0}
$$

such that

(1) $\rho_{t}^{c}:=e^{-\epsilon(t)} \rho_{t}$ holomorphic with respect to $J_{\epsilon(t)}$,

(2) $\rho_{K}$ is $\partial_{H}$-exact and $\bar{\partial}_{H}^{*}$-closed, $(|K| \geq 1)$

(3) $\rho_{t}$ converges with radius $\frac{1}{16 a^{2} c^{2}}$.

Proof. In step 1, we construct $\rho_{t}$, in step 2, we prove $\rho_{t}$ is $L^{2}$ convergent in $t<\frac{1}{16 a^{2} c^{2}}$, and in step 3 , we prove $\rho_{t}$ is smooth.

Step 1: We shall construct $\rho_{t}$.

If $\rho_{t}^{c}:=e^{-\epsilon(t)} \rho_{t}$ is holomorphic with respect to $J_{\epsilon(t)}$, by Proposition 6.1 . we have

$$
\bar{\partial}_{H} \rho_{t}=\partial_{H}\left(\epsilon(t) \rho_{t}\right) .
$$

Let

$$
\begin{aligned}
\rho_{t} & :=\rho_{0}+\sum_{k \geq 1} \sum_{i_{1}+\cdots+i_{N}=k} \rho_{i_{1} \cdots i_{N}}\left(t^{1}\right)^{i_{1}} \cdots\left(t^{N}\right)^{i_{N}} \in U_{0, M}, \\
\epsilon(t) & :=\sum_{k \geq 1} \sum_{i_{1}+\cdots+i_{N}=k} \epsilon_{i_{1} \cdots i_{N}}\left(t^{1}\right)^{i_{1}} \cdots\left(t^{N}\right)^{i_{N}} \in \wedge^{2} E^{*} .
\end{aligned}
$$


Some Results of Deformations on Compact $H$-twisted Generalized...163

Comparing the coefficients of $t=\left(t^{1}, \cdots, t^{N}\right)$, we have

$$
\begin{aligned}
& k=0, \bar{\partial}_{H} \rho_{0}=0, \\
& k \geq 1, \bar{\partial}_{H} \rho_{i_{1} \cdots i_{N}}=\partial_{H}\left(\sum_{j_{\alpha}+h_{\alpha}=i_{\alpha}, j_{\alpha}, h_{\alpha} \geq 0} \epsilon_{j_{1} \cdots j_{N}} \rho_{h_{1} \cdots h_{N}}\right) \cdot\left(i_{1}+\cdots+i_{N}=k\right)
\end{aligned}
$$

Let

$$
\eta_{i_{1} \cdots i_{N}}:=\partial_{H}\left(\sum_{j_{\alpha}+h_{\alpha}=i_{\alpha}, j_{\alpha}, h_{\alpha} \geq 0} \epsilon_{j_{1} \cdots j_{N}} \rho_{h_{1} \cdots h_{N}}\right) .
$$

We shall prove that $\bar{\partial}_{H} \eta_{i_{1} \cdots i_{N}}=0$ by induction on $i_{1}+\cdots+i_{N}=k$ and construct $\rho_{i_{1} \cdots i_{N}}$.

For $k=1$, we have

$$
\begin{aligned}
\bar{\partial}_{H} \eta_{i} & :=\bar{\partial}_{H} \partial_{H}\left(\epsilon_{i} \rho_{0}\right) \\
& =-\partial_{H} \bar{\partial}_{H}\left(\epsilon_{i} \rho_{0}\right) \\
& =-\partial_{H}\left(d_{E} \epsilon_{i}\right) \rho_{0} \\
& =0 .
\end{aligned}
$$

So by Proposition 4.2 , the equation

$$
\bar{\partial}_{H} \rho_{i}=\partial_{H}\left(\epsilon_{i} \rho_{0}\right)
$$

has the unique solution

$$
\rho_{i}=\bar{\partial}_{H}^{*} G \partial_{H}\left(\epsilon_{i} \rho_{0}\right)
$$

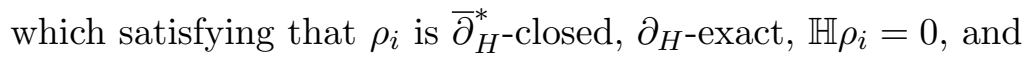

$$
\left\|\rho_{i}\right\|^{2} \leq\left(\partial_{H}\left(\epsilon_{i} \rho_{0}\right), G \partial_{H}\left(\epsilon_{i} \rho_{0}\right)\right)
$$


If we suppose that for $i_{1}+\cdots+i_{N} \leq k-1, \bar{\partial}_{H} \eta_{i_{1} \cdots i_{N}}=0$ holds and $\rho_{i_{1} \cdots i_{N}}$ is constucted. For $i_{1}+\cdots+i_{N}=k$, we have

$$
\begin{aligned}
& \bar{\partial}_{H} \eta_{i_{1} \cdots i_{N}}:=\bar{\partial}_{H} \partial_{H}\left(\sum_{j_{\alpha}+h_{\alpha}=i_{\alpha}, j_{\alpha}, h_{\alpha} \geq 0} \epsilon_{j_{1} \cdots j_{N}} \rho_{h_{1} \cdots h_{N}}\right) \\
& =-\partial_{H} \bar{\partial}_{H}\left(\sum_{j_{\alpha}+h_{\alpha}=i_{\alpha}, j_{\alpha}, h_{\alpha} \geq 0} \epsilon_{j_{1} \cdots j_{N}} \rho_{h_{1} \cdots h_{N}}\right) \\
& =-\partial_{H} \sum_{j_{\alpha}+h_{\alpha}=i_{\alpha}}\left(\left(d_{E} \epsilon_{j_{1} \cdots j_{N}}\right) \rho_{h_{1} \cdots h_{N}}+\epsilon_{j_{1} \cdots j_{N}}\left(\bar{\partial}_{H} \rho_{h_{1} \cdots h_{N}}\right)\right) \\
& =-\partial_{H} \sum_{j_{\alpha}+h_{\alpha}=i_{\alpha}}\left(-\sum_{l_{\alpha}+p_{\alpha}=j_{\alpha}} \frac{1}{2}\left[\epsilon_{l_{1} \cdots l_{N}}, \epsilon_{p_{1} \cdots p_{N}}\right]_{H} \rho_{h_{1} \cdots h_{N}}\right. \\
& \left.+\sum_{l_{\alpha}+p_{\alpha}=h_{\alpha}} \epsilon_{j_{1} \cdots j_{N}} \partial_{H}\left(\epsilon_{l_{1} \cdots l_{N}} \rho_{p_{1} \cdots p_{N}}\right)\right) \\
& =\frac{1}{2} \partial_{H} \sum_{l_{\alpha}+p_{\alpha}+h_{\alpha}=i_{\alpha}}\left(-\partial_{H}\left(\epsilon_{l_{1} \cdots l_{N}} \epsilon_{p_{1} \cdots p_{N}} \rho_{h_{1} \cdots h_{N}}\right)\right. \\
& +\epsilon_{l_{1} \cdots l_{N}} \partial_{H}\left(\epsilon_{p_{1} \cdots p_{N}} \rho_{h_{1} \cdots h_{N}}\right)+\epsilon_{p_{1} \cdots p_{N}} \partial_{H}\left(\epsilon_{l_{1} \cdots l_{N}} \rho_{h_{1} \cdots h_{N}}\right) \\
& -\partial_{H}\left(\sum_{l_{\alpha}+p_{\alpha}=h_{\alpha}} \epsilon_{j_{1} \cdots j_{N}} \partial_{H}\left(\epsilon_{l_{1} \cdots l_{N}} \rho_{p_{1} \cdots p_{N}}\right)\right) \\
& =\partial_{H} \sum_{l_{\alpha}+p_{\alpha}+h_{\alpha}=i_{\alpha}}+\epsilon_{l_{1} \cdots l_{N}} \partial_{H}\left(\epsilon_{p_{1} \cdots p_{N}} \rho_{h_{1} \cdots h_{N}}\right) \\
& -\partial_{H}\left(\sum_{l_{\alpha}+p_{\alpha}=h_{\alpha}} \epsilon_{j_{1} \cdots j_{N}} \partial_{H}\left(\epsilon_{l_{1} \cdots l_{N}} \rho_{p_{1} \cdots p_{N}}\right)\right) \\
& =0 \text {. }
\end{aligned}
$$

The 3rd equality holds since the definition of Lie derivation $d_{E}$; the 4 th equality is by induction and using the integral condition of $\epsilon(t)$; the 5th equality holds since Lemma 3.2 and each $\epsilon_{i_{1} \cdots i_{N}} \in \wedge^{2} E^{*}$; and the 6th equality holds since $\partial_{H}^{2}=0$.

From the discussion above, we know that the conditions of Proposition 4.2 hold. So we have the equation

$$
\bar{\partial}_{H} \rho_{i_{1} \cdots i_{N}}=\partial_{H}\left(\sum_{j_{\alpha}+h_{\alpha}=i_{\alpha}, j_{\alpha}, h_{\alpha} \geq 0} \epsilon_{j_{1} \cdots j_{N}} \rho_{h_{1} \cdots h_{N}}\right)
$$

has the unique solution

$$
\rho_{i_{1} \cdots i_{N}}=\bar{\partial}_{H}^{*} G \partial_{H}\left(\sum_{j_{\alpha}+h_{\alpha}=i_{\alpha}, j_{\alpha}, h_{\alpha} \geq 0} \epsilon_{j_{1} \cdots j_{N}} \rho_{h_{1} \cdots h_{N}}\right) \in U_{0, M} .
$$


Some Results of Deformations on Compact $H$-twisted Generalized...165

which satisfying that $\rho_{i_{1} \cdots i_{N}}$ is $\bar{\partial}_{H}^{*}$-closed, $\partial_{H}$-exact, $\mathbb{H} \rho_{i_{1} \cdots i_{N}}=0$, and

$$
\left\|\rho_{i_{1} \cdots i_{N}}\right\|^{2} \leq\left(\partial_{H}\left(\sum_{j_{\alpha}+h_{\alpha}=i_{\alpha}} \epsilon_{j_{1} \cdots j_{N}} \rho_{h_{1} \cdots h_{N}}\right), G \partial_{H}\left(\sum_{j_{\alpha}+h_{\alpha}=i_{\alpha}} \epsilon_{j_{1} \cdots j_{N}} \rho_{h_{1} \cdots h_{N}}\right)\right) .
$$

Step 2: We show $\rho_{t}$ is $L^{2}$ converges at $t<\frac{1}{16 a^{2} c^{2}}$.

We have already known that

$$
\begin{aligned}
& \sum_{k_{1}+\cdot+k_{N}=k}\left\|\rho_{k_{1} \cdots k_{N}}\right\| \\
:= & \sum_{k_{1}+\cdot+k_{N}=k}\left\|\bar{\partial}_{H}^{*} G \partial_{H}\left(\sum_{j_{\alpha}+h_{\alpha}=k_{\alpha}, j_{\alpha}, h_{\alpha} \geq 0} \epsilon_{j_{1} \cdots j_{N}} \rho_{h_{1} \cdots h_{N}}\right)\right\| \\
\leq & (2 c) \sum_{\left.k_{j_{1} \cdots j_{N}} \rho_{h_{1} \cdots h_{N}}\right) \|}\left\|\sum_{k_{1}+\cdot+k_{N}=k} \sum_{j_{\alpha}+h_{\alpha}=k_{\alpha}, j_{\alpha}, h_{\alpha} \geq 0}(2 c)\right\| \epsilon_{j_{1} \cdots j_{N}}\left\|_{s+\alpha} \cdot\right\| \rho_{h_{1} \cdots h_{N}} \| .
\end{aligned}
$$

The 1st inequality holds since Lemma 4.1, and the 2nd inequality holds by the definition of the Hölder norms.

Also we set $\left\{x_{i}\right\}_{i=1}^{\infty}$, such that $x_{1}=2 a^{2} c, x_{k}=(2 c) \sum_{i=1}^{k-1} x_{i} x_{k-i}$ just as in Lemma 3.4. As we assume the existence of $\epsilon(t)$ which is constructed in Theorem 5.1, and we also assume that $x_{k} \geq \sum_{k_{1}+\cdots+k_{N}=k}\left\|\epsilon_{k_{1} \cdots k_{N}} \rho_{0}\right\|_{s+\alpha}$. Next, we will show

$$
x_{k} \geq \sum_{k_{1}+\cdots+k_{N}=k}\left\|\rho_{k_{1} \cdots k_{N}}\right\|
$$

by induction on $k$.

For $k=1$, we have

$$
\begin{aligned}
x_{1} & \geq(2 c)\left(\sum_{i=1}^{N}\left\|\epsilon_{i}\right\|_{s+\alpha}\right)\left\|\rho_{0}\right\| \\
& \geq \sum_{i=1}^{N}\left\|\rho_{i}\right\| .
\end{aligned}
$$

If for $\leq k-1$, the inequality above holds, for $k$, we have 


$$
\begin{aligned}
x_{k} & :=(2 c) \sum_{i+j=k} x_{i} x_{j} \\
& \geq(2 c) \sum_{i+j=k}\left(\sum_{i_{1}+\cdots+i_{N}=i}\left\|\epsilon_{i_{1} \cdots i_{N}}\right\|_{s+\alpha}\right)\left(\sum_{j_{1}+\cdots+j_{N}=j}\left\|\rho_{j_{1} \cdots j_{N}}\right\|\right) \\
& \geq(2 c) \sum_{i_{1}+\cdots+k_{N}=k}\left\|\epsilon_{i_{1} \cdots i_{N}}\right\|_{s+\alpha} \cdot\left\|\rho_{j_{1} \cdots j_{N}}\right\| \\
& \sum_{i_{1}+\cdots+i_{N}+j_{1}+\cdots+j_{N}=k}^{\left\|\rho_{k_{1} \cdots k_{N}}\right\| .}
\end{aligned}
$$

The 1st inequality is the induction and the formula $(5.2)$, the in the 2 nd inequality we need that $\epsilon(t)$ has the convergence in 5.1 , and the last inequality holds since the formula (6.1).

Hence, we get

$$
\begin{aligned}
\left\|\rho_{t}\right\|: & \left\|\rho_{0}+\sum_{k \geq 1} \sum_{k_{1}+\cdots+k_{N}=k} \rho_{k_{1} \cdots k_{N}}\left(t^{1}\right)^{k_{1}} \cdots\left(t^{N}\right)^{k_{N}}\right\| \\
\leq & \left\|\rho_{0}\right\|+\sum_{k \geq 1} \sum_{k_{1}+\cdots+k_{N}=k}\left\|\rho_{k_{1} \cdots k_{N}}\left(t^{1}\right)^{k_{1}} \cdots\left(t^{N}\right)^{k_{N}}\right\| \\
\leq & a+(2 c) \sum_{k \geq 1, i_{1}+\cdots+i_{N}+j_{1}+\cdots+j_{N}=k}\left\|\epsilon_{i_{1} \cdots i_{N}}\right\|\left\|_{s+\alpha} \cdot\right\| \rho_{j_{1} \cdots j_{N}} \| \cdot|t|^{k} \\
& \left(t=\max _{i}\left\{t^{i}\right\}\right) \quad \sum_{k \geq 2, i_{1}+\cdots+i_{N}+j_{1}+\cdots+j_{N}=k}\left\|\epsilon_{i_{1} \cdots i_{N}}\right\|_{s+\alpha} \cdot\left\|\rho_{j_{1} \cdots j_{N}}\right\| \\
\leq & a+2 a^{2} c|t|+\left.(2 c)\right|_{k} ^{k} \\
\leq & a+x_{1}|t|+\sum_{k \geq 2} x_{k}|t|^{k} \\
< & +\infty
\end{aligned}
$$

at $|t| \leq \frac{1}{16 a^{2} c^{2}}$.

\section{Step 3:}

Since $\rho_{t}^{c}:=e^{-\epsilon(t)} \rho_{t}$ is holomorphic with respect to $J_{\epsilon(t)}$, we can get that

$$
\bar{\partial}_{H, t} \rho_{t}^{c}=0 .
$$

Since $\rho_{t}^{c}:=e^{-\epsilon(t)} \rho_{t} \in U_{0, t}$ is the canonical bundle in $M_{t}$, where

$$
\wedge^{*} T_{M_{t}}^{*}=U_{0, t} \oplus U_{1, t} \oplus \ldots \oplus U_{2 n, t}
$$


Some Results of Deformations on Compact $H$-twisted Generalized...167

is the decomposition in Definition 2.3, we can get that

$$
\bar{\partial}_{H, t}^{*} \rho_{t}^{c}=0
$$

by $\bar{\partial}_{H, t}^{*} \rho_{t}^{c} \in U_{-1, t}=0$.

So we get

$$
\triangle_{\bar{\partial}_{H, t}^{*}} \rho_{t}^{c}=0
$$

Thus, by the fact that $\triangle_{\bar{\partial}_{H, t}^{*}}$ is a strongly elliptic operator and the elliptic regularity theorem, we get that $\rho_{t}^{c}$ is smooth.

Proposition 6.5. We have

$$
\left[\rho_{t}^{c}\right]=\left[\rho_{0}\right]+\sum_{i=1}^{N}\left[\mathbb{H}\left(-\epsilon_{i} \rho_{0}\right)\right] t+O\left(|t|^{2}\right),
$$

where $\left[\rho_{0}\right]$ means a representation in $H_{\bar{\partial}_{H}}^{0}\left(M, \wedge^{*} T_{M}^{*}\right)$, and $O\left(|t|^{2}\right)$ denotes the terms in $H_{\bar{\partial}_{H}}^{4}\left(M, \wedge^{*} T_{M}^{*}\right) \oplus \cdots \oplus H_{\bar{\partial}_{H}}^{2 n}\left(M, \wedge^{*} T_{M}^{*}\right)$ of order at least 2 in $t$.

Proof. On convergence radius, we have

$$
\begin{aligned}
{\left[\rho_{t}^{c}\right]:=} & {\left[\rho_{0}\right]+\sum_{i=1}^{N}\left[\mathbb{H}\left(-\epsilon_{i} \rho_{0}\right)\right] t+\sum_{I,|I| \geq 2}\left[\mathbb{H}\left((-1)^{|I|} \epsilon_{I} \rho_{0}\right)\right] t^{|I|} } \\
& +\sum_{i, J,|J| \geq 1}\left[\mathbb{H}\left(-\epsilon_{i} \rho_{J}\right)\right] t^{1+|J|}+\sum_{I, J,|I| \geq 2,|J| \geq 1}\left[\mathbb{H}\left((-1)^{|I|} \epsilon_{I} \rho_{J}\right)\right] t^{|I|+|J|} \\
= & {\left[\rho_{0}\right]+\sum_{i=1}^{N}\left[\mathbb{H}\left(-\epsilon_{i} \rho_{0}\right)\right] t+O\left(|t|^{2}\right), }
\end{aligned}
$$

where $t^{I}:=\left(t_{1}\right)^{i_{1}} \ldots\left(t_{N}\right)^{i_{N}}, \epsilon_{I}:=\epsilon_{i_{1} \ldots t_{N}}$.

Remark 6.6. Here $-\epsilon_{i} \rho_{0}$ not necessary in $H_{\bar{\partial}_{H}}^{2}\left(M, \wedge^{*} T_{M}^{*}\right)$. In Calabi-Yau case, $-\epsilon_{i} \rho_{0} \in H_{\bar{\partial}_{H}}^{2}\left(M, \wedge^{*} T_{M}^{*}\right)$ and thus $\partial_{H}\left(-\epsilon_{i} \rho_{0}\right)=0$, all $\rho_{K}=0$ just as in Proposition 6.2. 


\section{References}

[1] D. Baraglia, Variation of Hodge structure for generalized complex manifolds, (2012) arXiv:math.DG/1205.0240v1.

[2] M. Gualtieri, Generalized complex geometry, D. Phil. thesis, Oxford University, (2003) arXiv:math.DG/0401221.

[3] M. Gualtieri, Generalized geometry and the Hodge decomposition, (2004) arXiv:math.DG/0409093v1.

[4] M. Gualtieri, 18.969 Topics in geometry (2006) http://www.math.toronto.edu/mgualt/gcg/geometrynotes.pdf.

[5] M. Gualtieri, Generalized Kähler geometry (2010) arXiv:1007.3485.

[6] M. Gualtieri, Generalized complex geometry (2007) arXiv:math/0703298.

[7] P. Griffiths and J. Harris, Principles of algebraic geometry, Pure and Applied Mathematics, Wiiey-interscience, New York (1978).

[8] F. Guan, A proof of generalized Kähler identities(in Chinese), (2008).

[9] R. Goto, On deformation of generalized Calabi-Yau, hyperKähler, $G_{2}$ and Spin(7) structures I, (2005) aiXiv:math/0512211v1.

[10] N. Hitchin, Gerneralized Calabi-Yau manifolds, Q.J. Math. 54(3) (2003) 281-308.

[11] K. Kodaira, Complex manifolds and deformation of complex structure, Springer-Verlag (1981).

[12] K. Liu, S. Rao, and X. Yang, Quasi-isometry and deformations of CalabiCYau manifolds,Inventiones Mathematicae, (2014) DOI 10.1007/s00222-014-0516-1, arXiv:math.DG/1207.1182v1.

[13] K. Liu and S. Rao, Remarks on the Cartan formula and its applications, Asian Journal of Mathematics, (2012) 157-170, arXiv:1104.1240v1.

[14] Y. Li, On deformation of generalized complex structures:the generalized Calabi-Yau case,(2005). arXiv:hep-th/0508030v2.

[15] J. Morrow and K. Kodaira, Complex manifolds, Holt, Rinehart and Winston, Inc., New York-Montreal, Que.-London,(1971).

[16] P. Ševera and A. Weinstein, Poisson geometry with a 3-form background, Prog. Theor. Phys. Suppl. 144, (2001) 145-154. 
Some Results of Deformations on Compact $H$-twisted Generalized...169

[17] N. Shimakura, Partial differential operators of elliptic type, Translated and revised from the (1978) Japanese original by the author, Translations of Mathematical Monographs, 99, American Mathematical Society, Providence, RI, (1992).

[18] G. Tian, Smoothness of the universal deformation space of compact Calabi-Yau manifolds and its Petersson-Weil metric, Mathematical aspects of string theory(San Diego, California, 1986). Advanced Series in Mathematical Physics, vol. 1, 629-646. World Scientific Publishing, Singapore(1987).

[19] A. Todorov, The Weil-Petersson geometry of the moduli space of $\mathbb{S U}(\mathrm{n} \geq 3)$ (Calabi-Yau) manifolds I. Commun. Math. Phys. 126(2), 325346(1989).

[20] K. Wei, Global canonical family of deformations of compact $H$-twisted Generalized Calabi-Yau manifolds.

[21] R. Wells, Differential analysis on complex manifolds, Springer-Verlag, 2nd edition (1980).

[22] Q. Zhao and S. Rao, Applications of deformation formula of holomorphic one-forms, Pacific Journal of Mathematics Vol. 266, No. 1, (2013)221-256.

Kang Wei

Center of Mathematical Sciences,

Zhejiang University,

Hangzhou, Zhejiang 310027,

China

E-mail: kangwei296@gmail.com, kang_wei5@hotmail.com 
